DOI 10.4171/JEMS/349

Matthias Hammerl · Petr Somberg · Vladimír Souček · Josef Šilhan

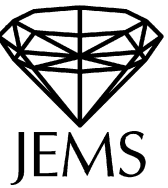

\title{
On a new normalization for tractor covariant derivatives
}

Received March 31, 2010 and in revised form August 21, 2011

\begin{abstract}
A regular normal parabolic geometry of type $G / P$ on a manifold $M$ gives rise to sequences $D_{i}$ of invariant differential operators, known as the curved version of the BGG resolution. These sequences are constructed from the normal covariant derivative $\nabla^{\omega}$ on the corresponding tractor bundle $V$, where $\omega$ is the normal Cartan connection. The first operator $D_{0}$ in the sequence is overdetermined and it is well known that $\nabla^{\omega}$ yields the prolongation of this operator in the homogeneous case $M=G / P$. Our first main result is the curved version of such a prolongation. This requires a new normalization of the tractor covariant derivative on $V$. Moreover, we obtain an analogue for higher operators $D_{i}$. In that case one needs to modify the exterior covariant derivative $d^{\nabla^{\omega}}$ by differential terms. Finally we illustrate these results with simple examples in projective, conformal and Grassmannian geometry. Our approach is based on standard BGG techniques.
\end{abstract}

Keywords. Parabolic geometry, prolongation of invariant overdetermined PDE's, BGG sequence, tractor covariant derivatives

\section{Introduction}

The problem how to find a prolongation of an overdetermined system of PDE's acting between sections of vector bundles is classical and has been studied for a long time. A systematic procedure to solve such problems was developed by D. C. Spencer (see [32]) and his coworkers. One of the tools employed by him was the Spencer resolution of the system, which is useful for description of many properties of solutions of the system. In particular, there is a class of systems of finite type whose solutions are determined by a finite jet at a chosen point. Spencer found a suitable characterization of such systems in his studies. His general results are quite useful but in specific examples, in particular for equations arising in a geometric context, a more efficient analysis can be obtained by employing techniques more adapted to the geometric structure.

M. Hammerl: Fakultät für Mathematik, Nordbergstraße 15, 1090 Wien, Austria; e-mail: matthias.hammerl@univie.ac.at

P, Somberg, V. Souček: Mathematical Institute, Sokolovska 83, Karlin, 180 00, Praha 8, Czech Republic; e-mail: somberg@karlin.mff.cuni.cz, soucek@karlin.mff.cuni.cz

J. Šilhan: Masaryk University, Janáčkovo nam. 2a, 66295 Brno, Czech Republic; e-mail: silhan@math.muni.cz

Mathematics Subject Classification (2010): 58J70, 53A55, 58A32, 53A20, 53A30 
Important examples of overdetermined systems can be found in many areas of geometry. Examples in conformal geometry include, e.g., Killing vectors, conformal Killing vectors, Killing-Yano forms, equations for Einstein scales, etc. (see [30, 19, 26]). In such cases, it was possible to get much better results, because the relevant manifolds were equipped with a rich geometric structure making it possible to use very efficient tools coming from representation theory. To illustrate this in more detail, let us recall that the most famous example of a resolution of an overdetermined system is the de Rham sequence for differential forms on a general manifold. The overdetermined system in this case is the gradient of a function. The de Rham differentials $d$ forming the resolution are distinguished by their invariance with respect to the group of diffeomorphisms acting on the manifold.

Many more explicit examples of overdetermined systems and their resolutions can be described in cases where the manifold is equipped with a richer geometric structure. Typical examples are manifolds with a given projective, conformal, quaternionic, or CR structure. Homogeneous models of such structures are given by homogeneous spaces $G / P$, where $G$ is a semisimple Lie group and $P$ a parabolic subgroup. On such spaces there exist infinite sequences of resolutions (analogues to the de Rham resolutions), one for each irreducible $G$-module. The de Rham resolution is the resolution for the trivial $G$-module. A feature of such resolutions is that operators forming the sequence are (typically) higher order operators (with orders rising with the complexity of the $G$-module). They are dual versions of the famous Bernstein-Gel'fand-Gel'fand resolutions of irreducible $G$-modules by Verma modules found in the 70's in representation theory. Following ideas of É. Cartan, it is possible to introduce 'curved versions' of such homogeneous models known under the name of parabolic geometries (see [8]). Curved versions of such resolutions were constructed recently in complete generality in $[9,5]$. They are again formed by invariant differential operators, but their composition is now nontrivial due to nontrivial curvature of general curved structures.

To be more specific, let us now recall more details on parabolic geometries. Let $G$ be a (real) semisimple Lie group and $P$ its parabolic subgroup. Following ideas of É. Cartan, the homogeneous space $G / P$ is a flat model for a curved parabolic geometry of type $(G, P)$, which is specified by a couple $(\mathcal{G}, \omega)$, where $\mathcal{G} \rightarrow M$ is a principal $P$-bundle and $\omega$ is a Cartan connection. It is well known that such a geometry can be characterized by an underlying geometric structure on the manifold $M$, together with suitable conditions applied to the Cartan connection needed to remove ambiguities in its definition. A key condition is a normalization condition expressed using the language of cohomology of Lie algebras. Cartan connections satisfying this normalization condition are called normal Cartan connections. To have an equivalence of categories between the category of parabolic structures $(\mathcal{G}, \omega)$ on $M$ and the underlying geometric structure on $M$, it is necessary to add an additional technical condition on $\omega$ (called regularity). Full details on this correspondence can be found in [8].

Distinguished examples of this procedure are the normal Cartan connections constructed for a conformal structure by É. Cartan and for a CR structure by Chern and Moser $([10,12])$. Let us consider a regular normal parabolic geometry $(\mathcal{G}, \omega)$ of type $(G, P)$. For any $G$-module $\mathbb{V}$, the tractor bundle $V$ over $M$ is (by definition) the vec- 
tor bundle associated to $\mathcal{G}$ and the representation $\mathbb{V}$ (restricted to $P$ ). The normal Cartan connection $\omega$ on $\mathcal{G}$ then induces the tractor covariant derivative $\nabla^{\omega}$ on $V$, which is then used in various problems in analysis and/or geometry on $M$ (e.g., to construct differential invariants on the corresponding parabolic geometry). For example, it plays the key role in the construction of Bernstein-Gel'fand-Gel'fand (BGG) sequences of invariant differential operators (see $[9,5])$ and prolongation procedures for first operators in BGG sequences (see e.g. [4]).

In particular, there is a lot of interest in the study of properties of the first operators in the BGG sequences, or their semilinear versions. Ideas behind the construction of these operators by the BGG machinery can be helpful in such problems. The construction uses tractor covariant derivatives acting on tractor bundles and suitable splitting operators (for details, see Sect. 3). In some simple cases there is a one-to-one correspondence between solutions of the first BGG equation and the kernel of the corresponding tractor covariant derivative. In other words, the tractor covariant derivative is the prolongation of the first BGG operator. But such a simple correspondence is not valid in general.

A general scheme for prolongation of the first BGG operator for parabolic geometries with commutative nilpotent radical was introduced in [4]. The authors not only treat the prolongation for linear overdetermined systems with a particular behavior of the symbol but they also allow semilinear systems having the same symbol as in the linear case and allowing general nonlinear behavior of the lower order part of the operator. A generalization to contact cases can be found in [16] and an extension to general parabolic geometries is discussed in [28]. The procedure used in [4] is efficient but not invariant. In quite a few special cases (see $[6,13,17,15,21,19]$ ), several authors found an invariant way to compute a deformation of the normal tractor covariant derivative having the property that its kernel can be identified with solutions of the first BGG sequence.

The new normalization of tractor covariant derivatives developed in this paper is motivated by a wish to extend these examples to a general scheme. We shall study the problem of a suitable normalization for tractor covariant derivatives for a general parabolic geometry in a systematic way and show that there is a distinguished alternative of the usual normalization of tractor covariant derivatives on tractor bundles giving directly a canonical prolongation of the first BGG operator in an invariant way.

The normal tractor covariant derivative is induced from the normal Cartan connection on the principal bundle $\mathcal{G}$. An important observation is that if we want to find a covariant derivative on tractor bundles giving an invariant prolongation of the first BGG operator, it is necessary to adapt (in contrast to $\nabla^{\omega}$ ) the normalization condition to the choice of the tractor bundle under consideration.

The main results of the paper can be described as follows. Let us consider a regular normal parabolic geometry of type $(G, P)$ given by the couple $(\mathcal{G}, \omega)$. To any irreducible $G$-module $\mathbb{V}$, there is associated the covariant derivative $\nabla^{\omega}$ on the associated vector bundle $V$. The space of all covariant derivatives on $V$ is the affine space modeled on the vector space $\mathcal{E}^{1}$ (End $V$ ). We want to find a deformation of $\nabla^{\omega}$ by $\Phi \in \mathcal{E}^{1}$ (End $V$ ) satisfying a new normalization condition (adapted to the choice of $\mathbb{V}$ ) in such a way that the resulting covariant derivative will have suitable properties. 
The deformation $\Phi$ cannot be chosen arbitrarily. Firstly, the construction of the BGG sequence leads to the requirement of preserving the lowest homogeneous component of $\nabla^{\omega}$ (having homogeneity zero), hence we shall restrict to $\Phi \in \mathcal{E}^{1}$ (End $\left.V\right)^{1}$, where the superscript 1 indicates that $\Phi$ should have (total) homogeneity at least one. The desire to have good properties of the new covariant derivative in the prolongation procedure for the first BGG operator imposes further restrictions on the choice of $\Phi$. They will be expressed by properties of values of $\Phi(s) \in \mathcal{E}^{1}(V)$, where $s$ is a section of $V$. This leads to the following class of covariant derivatives on the tractor bundle $V$.

Definition 1.1. Let $\omega$ be the regular normal Cartan connection on the principal bundle $\mathcal{G}$ and let $\nabla^{\omega}$ be the associated covariant derivative on the associated vector bundle $V$. The class $\mathcal{C}$ of admissible covariant derivatives on $V$ is defined by

$$
\mathcal{C}=\left\{\nabla=\nabla^{\omega}+\Phi \mid \Phi \in \operatorname{Im}\left(\partial_{V}^{*} \otimes \operatorname{Id}_{V^{*}}\right), \Phi \in \mathcal{E}^{1}(\text { End } V)^{1}\right\}
$$

where $\partial_{V}^{*}$ is the Kostant differential corresponding to homology of $\mathfrak{g}_{\text {- }}$ with values in $\mathbb{V}$ (cf. [25]).

The condition $\Phi \in \operatorname{Im}\left(\partial_{V}^{*} \otimes \operatorname{Id}_{V^{*}}\right)$ is equivalent to $\Phi(s) \in \operatorname{Im} \partial_{V}^{*} \subset \mathcal{E}^{1}(V)$ for all $s \in \Gamma(V)$, where $\Gamma(V)$ denotes the space of sections of $V$.

The main theorem of the paper is then

Theorem 1.2. There exists a unique covariant derivative $\nabla \in \mathcal{C}$ such that

$$
\left(\partial_{V}^{*} \otimes \operatorname{Id}_{V^{*}}\right)\left(R^{\nabla}\right)=0,
$$

where $R^{\nabla} \in \mathcal{E}^{2}($ End $V)$ is the curvature of $\nabla$. Again, the condition $\left(\partial_{V}^{*} \otimes \operatorname{Id}_{V^{*}}\right)\left(R^{\nabla}\right)=0$ can be equivalently expressed as $\partial_{V}^{*}\left(R^{\nabla}(s)\right)=0$ for all sections $s$ of $V$.

The new covariant derivative $\nabla$ constructed in Theorem 1.2 gives a prolongation of the first BGG operator, hence we shall call the covariant derivative satisfying this new normalization condition the prolongation covariant derivative. The next main result is the theorem stating this property.

Theorem 1.3. Let us consider a parabolic geometry $(\mathcal{G}, \omega)$ modeled on a couple $(G, P)$. There is a one-to-one correspondence between the kernel of the first BGG operator for a $G$-module $\mathbb{V}$ and the kernel of the prolongation covariant derivative on the associated bundle $V$ over $M$.

In Section 4, we extend the previous construction to other operators in the BGG sequence. In these cases, we have to consider a more general deformation of the exterior derivative $d^{\nabla}$ by adding a differential term (instead of just an algebraic one, which was sufficient for the first operator in the BGG sequence).

Finally, we compare the general procedure developed in this paper with particular results obtained in some special cases and compute some other examples of prolongation covariant derivatives. They come from projective and Grassmannian geometry. 


\section{Normalization of tractor covariant derivatives}

\subsection{The double filtration on End $\mathbb{V}$}

Let $G$ be a semisimple Lie group (real or complex) and $P$ a parabolic subgroup of $G$. The choice of $P$ induces a grading $\mathfrak{g}=\bigoplus_{i=-k}^{k} \mathfrak{g}_{i}$ on the Lie algebra of $G$ : there is a grading element $E$ in $\mathfrak{g}_{0}$ acting by $i$ on $\mathfrak{g}_{i}$.

Every irreducible module $\mathbb{V}$ for $G$ is also graded by the action of $E$ as follows:

$$
\mathbb{V}=\bigoplus_{a \in A} \mathbb{V}_{a}, \quad \mathbb{V}^{*}=\bigoplus_{b \in A} \mathbb{V}_{-b}^{*}
$$

where $A$ is the set of all eigenvalues of $E$ on $\mathbb{V}$. A similar decomposition of $\mathfrak{g}_{+}$is given by $\mathfrak{g}_{+}=\mathfrak{g}_{1} \oplus \cdots \oplus \mathfrak{g}_{k}$.

The representation End $\mathbb{V} \simeq \mathbb{V} \otimes \mathbb{V}^{*}$ has the standard 'diagonal' grading induced by the action of $E$, given by

$$
\text { End } \mathbb{V}=\bigoplus_{\ell}(\text { End } \mathbb{V})_{\ell}, \quad(\text { End } \mathbb{V})_{\ell}:=\bigoplus_{a-b=\ell ; a, b \in A} \mathbb{V}_{a} \otimes \mathbb{V}_{-b}^{*} .
$$

The key point for the iterative process below is to consider the second 'vertical' grading on the product $\mathbb{V} \otimes \mathbb{V}^{*}$ by keeping the grading on $\mathbb{V}$ and using the trivial grading on $\mathbb{V}^{*}$. Hence the vertical grading is given by

$$
\text { End } \mathbb{V}=\bigoplus_{a \in A}(\text { End } \mathbb{V})_{a}, \quad(\text { End } \mathbb{V})_{a}:=\mathbb{V}_{a} \otimes \mathbb{V}^{*}
$$

The gradings are not $P$-invariant. We shall hence consider filtrations induced by the gradings above. For the diagonal grading, we shall define the filtration by

$$
(\text { End } \mathbb{V})^{\ell}=\bigoplus_{k \geq \ell}(\text { End } \mathbb{V})_{k}
$$

In particular, $(\text { End } \mathbb{V})^{1}$ always denotes the corresponding component with respect to the diagonal filtration.

For the vertical grading, the filtration is defined by

$$
(\text { End } \mathbb{V})^{a}=\bigoplus_{b \geq a}(\text { End } \mathbb{V})_{b}
$$

The grading of $\mathfrak{g}_{+}$also gives the standard filtration $\mathfrak{g}^{k} \subset \cdots \subset \mathfrak{g}^{1}=\mathfrak{g}_{+}$.

These filtrations (together with the filtration on $\mathfrak{g}_{+}$) also induce the filtrations on the chain spaces $\Lambda^{j}\left(\mathfrak{g}_{+}\right) \otimes$ End $\mathbb{V}$ for the Lie algebra homology and cohomology complexes. The differentials in the Lie algebra (co)homology of $\mathfrak{g}_{-}$with values in $\mathfrak{g}$-modules $\mathbb{W}$ are the maps $\partial \mathbb{W}: \Lambda^{j}\left(\mathfrak{g}_{+}\right) \otimes \mathbb{W} \rightarrow \Lambda^{j+1}\left(\mathfrak{g}_{+}\right) \otimes \mathbb{W}$, resp. $\partial_{\mathbb{W}}^{*}: \Lambda^{j}\left(\mathfrak{g}_{+}\right) \otimes \mathbb{W} \rightarrow$ $\Lambda^{j-1}\left(\mathfrak{g}_{+}\right) \otimes \mathbb{W}$. If $\mathbb{W}=$ End $\mathbb{V} \simeq \mathbb{V} \otimes \mathbb{V}^{*}$ for a $\mathfrak{g}$-module $\mathbb{V}$, we shall denote the operators $\partial_{\mathbb{V}} \otimes \operatorname{Id}_{\mathbb{V} *}$, resp. $\partial_{\mathbb{V}}^{*} \otimes I_{\mathbb{V}^{*}}$, simply by $\partial \mathbb{V}$, resp. $\partial_{\mathbb{V}}^{*}$. This should not lead to any confusion. 
The definition of $\partial_{\mathbb{V}}$ and $\partial_{\mathbb{V}}^{*}$ implies immediately that they preserve both the vertical and diagonal gradings on $\Lambda^{j}\left(\mathfrak{g}_{+}\right) \otimes$ End $\mathbb{V}$. Hence they respect both vertical and diagonal filtrations on $\Lambda^{j}\left(\mathfrak{g}_{+}\right) \otimes$ End $\mathbb{V}$. Below we shall use the induced operators between the graded bundles associated to the vertical filtration and we shall denote them by gr $\partial_{\mathbb{V}}$, resp. gr $\partial_{\mathbb{V}}^{*}$.

\subsection{Induced operators on associated graded bundles}

The spaces of $j$-forms on $M$ with values in a bundle $W$ will be denoted by $\mathcal{E}^{j}(W)$. They are isomorphic to the bundle induced by the $P$-module $\Lambda^{j}\left(\mathfrak{g}_{+}\right) \otimes \mathbb{W}$. Similarly, the tangent bundle is isomorphic to the bundle associated to the $P$-module $\mathfrak{g} / \mathfrak{p}$. All filtrations mentioned above are $P$-invariant and so they induce the corresponding filtrations on $\mathcal{E}^{j}$ (End $\left.V\right)$. We shall need, in particular, the diagonal filtrations $\mathcal{E}^{j}$ (End $\left.V\right)^{\ell}$, resp. the vertical filtration $\mathcal{E}^{j}(\text { End } V)^{a}$, induced on $\mathcal{E}^{j}($ End $V)$. We shall denote by $\operatorname{gr}_{\ell}\left(\mathcal{E}^{j}(\right.$ End $V)$ ), resp. $\operatorname{gr}_{a}\left(\mathcal{E}^{j}(\right.$ End $\left.V)\right)$ the associated graded bundles.

The operators gr $\partial_{\mathbb{V}}^{*}$ and gr $\partial \mathbb{V}$ are $P$-equivariant, hence they induce well defined maps $\partial_{V}^{*}$, resp. $\partial_{V}$, between the corresponding associated graded bundles.

We shall denote by gr $\partial_{V}$, resp. gr $\partial_{V}^{*}$, the direct sum of all maps $\operatorname{gr}_{a} \partial_{V}$, resp. $\operatorname{gr}_{a} \partial_{V}^{*}$, acting on the direct sum gr $\mathcal{E}^{j}($ End $V):=\bigoplus_{a} \operatorname{gr}_{a}\left(\mathcal{E}^{j}(\right.$ End $\left.V)\right)$. The operators gr $\partial_{V}$ and $\operatorname{gr} \partial_{V}^{*}$ then have the usual properties of the Kostant differentials. In particular, they are dual to each other (with respect to a suitable scalar product), which implies the usual properties of their kernels and images (Hodge decomposition).

Note also that $\mathcal{E}^{j}(V) \otimes V^{*}=\mathcal{E}^{j}($ End $V)$. Hence the standard filtration on $\mathcal{E}^{j}(V)$ is transferred (by the tensor product with $V^{*}$ ) to the vertical grading on $\mathcal{E}^{j}$ (End $V$ ). As an immediate corollary, $\varphi \in \mathcal{E}^{j}(\text { End } V)^{a}$ if and only if $\varphi s \in \mathcal{E}^{j}(V)^{a}$ for all sections $s \in \mathcal{E}^{0}(V)$.

\subsection{A choice of normalization}

Let us consider a regular parabolic geometry $(\mathcal{G}, \omega)$ over $M$ with the homogeneous model given by a couple $(G, P)$. For an irreducible $G$-module $\mathbb{V}$, we shall consider the associated tractor bundle $V$ on $M$. The curvature $\kappa$ of the Cartan connection $\omega$ is a two-form with values in the adjoint tractor bundle $\mathcal{A} \simeq \mathcal{G} \times{ }_{P} \mathfrak{g}$. The usual normalization condition for $\omega$, expressed in terms of the Kostant differential $\partial^{*}$ corresponding to homology of $\mathfrak{g}_{-}$ with values in $\mathfrak{g}$, requires the curvature $\kappa$ to be $\partial^{*}$-closed. In terms of the associated covariant derivative $\nabla^{\omega}$ on $V$, the curvature $R^{\nabla^{\omega}}$ of $\nabla^{\omega}$ is a two-form with values in End $V$ and the normalization condition can be expressed using the Kostant differential $\partial^{*}$ for End $V$ as

$$
\partial^{*}\left(R^{\nabla^{\omega}}\right)=0 .
$$

Given a choice of the bundle $V$, we are going to change the normalization condition for a covariant derivative $\nabla$ on $V$. Let $\operatorname{Id}_{V^{*}}$ denote the identity map on $V^{*}$. As above in the algebraic version, we shall consider the operators

$$
\partial_{V} \otimes \operatorname{Id}_{V^{*}}, \quad \partial_{V}^{*} \otimes \operatorname{Id}_{V^{*}}
$$


acting on $\mathcal{E}^{j}\left(\right.$ End $V$ ), forms with values in End $V \simeq V \otimes V^{*}$. Abusing the notation, we shall denote them by $\partial_{V}$, resp. $\partial_{V}^{*}$. It will always be clear whether the differentials act on forms with values in $V$ or forms with values in End $V$.

We shall now introduce a new normalization for covariant derivatives on $V$.

Definition 2.1. We shall call a covariant derivative $\nabla \in \mathcal{C}$ the prolongation covariant derivative if

$$
\partial_{V}^{*}\left(R^{\nabla}\right)=0
$$

where $R^{\nabla} \in \mathcal{E}^{2}($ End $V$ ) is the curvature of $\nabla$.

The choice of the name should suggest that the new normalization condition gives better properties to $\nabla$ in the prolongation procedure for the first operator in the BGG sequence corresponding to the representation $\mathbb{V}$.

We shall need the following property.

Lemma 2.2. If $\varphi \in \mathcal{E}^{1}(\text { End } V)^{a}$ and $\tau \in \mathcal{E}^{1}(V)$, then

$$
\varphi \wedge \tau \in \mathcal{E}^{2}(V)^{a+1} \text {. }
$$

Proof. Indeed, we can decompose $\varphi$ into homogeneous components

$$
\varphi=\sum_{j} \alpha_{j} \otimes v_{j} \otimes w_{j}, \quad \alpha_{j} \in \mathcal{E}^{1}, \quad v_{j} \in V, w_{j} \in V^{*},
$$

where the sum of the homogeneities of $\alpha_{j}$ and $v_{j}$ is greater than or equal to $a$. If we also decompose $\tau$ as

$$
\tau=\sum_{k} \beta_{k} \otimes u_{k}, \quad \beta_{k} \in \mathcal{E}^{1}, u_{k} \in V
$$

then the expression

$$
\varphi \wedge \tau=\sum_{j, k} w_{j}\left(u_{k}\right) \alpha_{j} \wedge \beta_{k} \otimes v_{j}
$$

clearly has summands of homogeneity greater than or equal to $a+1$.

\subsection{The main lemma}

The key information for the normalization procedure is the following fact concerning the induced change of curvature.

Lemma 2.3. Let $\nabla_{1}$, resp. $\nabla_{2}$, be two covariant derivatives from $\mathcal{C}$ related to each other by the deformation $\Phi=\nabla_{2}-\nabla_{1} \in \mathcal{E}^{1}(\text { End } V)^{1}$ and let $R_{1}$, resp. $R_{2}$, be the corresponding curvatures. If $\Phi \in \mathcal{E}^{1}(\text { End } V)^{a}$, then $R_{2}-R_{1} \in \mathcal{E}^{2}(\text { End } V)^{a}$ and

$$
\operatorname{gr}_{a}\left(R_{2}-R_{1}\right)=\left(\operatorname{gr}_{a} \partial_{V}\right)\left(\operatorname{gr}_{a} \Phi\right)
$$


Proof. Let $\omega$ be the normal Cartan connection for the chosen parabolic geometry and $\nabla$ the associated covariant derivative. It is well known that $\nabla$ and $d^{\nabla}$ preserve the standard filtration on $\mathcal{E}^{j}(V)$ and that the corresponding graded version of $\nabla$, resp. $d^{\nabla}$, is equal to gr $\partial_{V}$. A shift of $\nabla$ by $\Phi \in \mathcal{E}^{1}$ (End $\left.V\right)^{1}$ does not change this property, the same being true for $d^{\nabla+\Phi}$.

The change in curvature is then

$$
R_{2}-R_{1}=d^{\nabla} \Phi+[\Phi, \Phi]
$$

The result clearly belongs to $\mathcal{E}^{2}(\text { End } V)^{a}$, because the operator $d^{\nabla}$ preserves the filtrations and we can use Lemma 2.2 for the second term.

Then we get, for any $s \in \mathcal{E}^{0}(V)$,

$$
\begin{aligned}
\operatorname{gr}_{a}\left(\left(d^{\nabla} \Phi+[\Phi, \Phi]\right) s\right) & =\operatorname{gr}_{a}\left(\left(d^{\nabla} \Phi\right) s\right)=\operatorname{gr}_{a}\left(d^{\nabla}(\Phi s)-\Phi \wedge(\nabla s)\right) \\
& =\operatorname{gr}_{a}\left(\partial_{V}(\Phi(s))\right)=\left(\operatorname{gr}_{a} \partial_{V}\right)\left(\operatorname{gr}_{a}(\Phi(s))\right) .
\end{aligned}
$$

\subsection{Existence and uniqueness of the prolongation covariant derivative}

We now show the main theorem of this article:

Theorem 2.4. For each irreducible $G$-module $\mathbb{V}$, there exists a unique prolongation covariant derivative $\nabla \in \mathcal{C}$, i.e., a unique $\nabla \in \mathcal{C}$ such that

$$
\partial_{V}^{*}\left(R^{\nabla}\right)=0 .
$$

Proof. The curvature function of the regular normal connection $\omega$ for the corresponding parabolic geometry belongs (by definition of regularity) to $\mathcal{E}^{2}(\mathcal{A})^{1}$, so $R^{\nabla^{\omega}} \in \mathcal{E}^{2}(\text { End } V)^{1}$, and $\partial_{V}^{*}\left(R^{\nabla^{\omega}}\right) \in \mathcal{E}^{1}$ (End $\left.V\right)^{1}$. Lemma 2.5 below now shows that we can start with $\nabla^{\omega}$ and obtain by induction a unique $\nabla \in \mathcal{C}$ satisfying (1).

Lemma 2.5. Suppose that there is a tractor covariant derivative $\nabla \in \mathcal{C}$ with

$$
\partial_{V}^{*}\left(R^{\nabla}\right) \in \mathcal{E}^{1}(\text { End } V)^{1} \cap \mathcal{E}^{1}(\text { End } V)^{a},
$$

where $a \in A$ is such that $a+1$ belongs to A. Then there exists

$$
\Phi \in \mathcal{E}^{1}(\text { End } V)^{1} \cap \mathcal{E}^{1}(\text { End } V)^{a} \cap \operatorname{Im}\left(\partial_{V}^{*} \otimes \operatorname{Id}_{V^{*}}\right)
$$

such that for $\tilde{\nabla}=\nabla+\Phi$, one has

$$
\partial_{V}^{*}\left(R^{\tilde{\nabla}}\right) \in \mathcal{E}^{1}(\text { End } V)^{1} \cap \mathcal{E}^{1}(\text { End } V)^{a+1} .
$$

Moreover, $\Phi$ is unique up to terms of homogeneity $a+1$. In particular, $\tilde{\nabla} \in \mathcal{C}$ satisfying (2) is unique up to modifications by elements in

$$
\mathcal{E}^{1}(\text { End } V)^{1} \cap \operatorname{Im}\left(\partial_{V}^{*} \otimes \operatorname{Id}_{V^{*}}\right) \cap \mathcal{E}^{1}(\text { End } V)^{a+1} .
$$


Proof. The spaces $\left\{\mathcal{E}^{1}(\operatorname{End} V)^{1} \cap \mathcal{E}^{1}(\operatorname{End} V)^{a}\right\}_{a=0}^{r}$ give a descending filtration of $\mathcal{E}^{1}(\text { End } V)^{1}$. The filtration is preserved by the maps $\partial_{V}$ and $\partial_{V}^{*}$, hence they induce maps on the associated graded bundle (for simplicity we denote them by the same symbols as for the full filtration of $\mathcal{E}^{1}$ (End $V$ )). The standard Kostant decomposition says that Ker gr $\partial_{V}^{*}$ and $\operatorname{Im} \operatorname{gr} \partial_{V}$ are complementary subspaces of the graded bundle gr $\mathcal{E}^{1}$ (End $\left.V\right)^{1}$. In particular, gr $\partial_{V}^{*}$ restricts to an isomorphism of $\operatorname{Im} \operatorname{gr} \partial_{V}$ and $\operatorname{Im} \operatorname{gr} \partial_{V}^{*}$.

Hence we can define $\varphi \in \operatorname{gr}_{a}\left(\mathcal{E}^{1}(\text { End } V)^{1}\right)$ by

$$
\varphi:=\square^{-1} \partial_{V}^{*}\left(R^{\tilde{\nabla}}\right)
$$

which then has the property that

$$
\left(\operatorname{gr} \partial_{V}^{*}\right)\left(\left(\operatorname{gr} \partial_{V}\right)(\varphi)\right)=\operatorname{gr}_{a}\left(\partial_{V}^{*}\left(R^{\nabla}\right)\right) .
$$

Let $\Phi \in \mathcal{E}^{1}$ (End $\left.V\right)^{1} \cap \mathcal{E}^{1}(\text { End } V)^{a} \cap \operatorname{Im}\left(\partial_{V}^{*} \otimes \operatorname{Id}_{V^{*}}\right)$ be a preimage of $\varphi$. Then we define a corrected covariant derivative by $\tilde{\nabla}:=\nabla-\Phi$.

Due to Lemma 2.3, we get

$$
\begin{aligned}
\operatorname{gr}_{a}\left(\partial_{V}^{*}\left(R^{\tilde{\nabla}}\right)\right) & =\operatorname{gr}_{a}\left(\partial_{V}^{*}\left(R^{\nabla}\right)\right)-\left(\operatorname{gr}_{V}^{*}\right)\left(\operatorname{gr}_{a}\left(R^{\nabla}-R^{\tilde{\nabla}}\right)\right) \\
& =\operatorname{gr}_{a}\left(\partial_{V}^{*}\left(R^{\nabla}\right)\right)-\left(\operatorname{gr}_{V}^{*}\right)\left(\left(\operatorname{gr}_{V}\right)\left(\operatorname{gr}_{a}(\Phi)\right)\right)=0 .
\end{aligned}
$$

Hence $\tilde{\nabla}$ has the required properties.

For the uniqueness up to terms of homogeneity higher than $a$, assume that we have another $\Phi^{\prime} \in \mathcal{E}^{1}(\text { End } V)^{1} \cap \mathcal{E}^{1}(\text { End } V)^{a} \cap \operatorname{Im}\left(\partial_{V}^{*} \otimes \operatorname{Id}_{V^{*}}\right)$ such that $\tilde{\nabla}^{\prime}=\nabla+\Phi^{\prime}$ satisfies $\partial_{V}^{*}\left(R^{\tilde{\nabla}^{\prime}}\right) \in \mathcal{E}^{1}(\text { End } V)^{a+1}$. Then $\Phi^{\prime}-\Phi$ belongs to $\mathcal{E}^{1}(\text { End } V)^{1} \cap \operatorname{Im} \partial_{V}^{*}$, and by assumption $\operatorname{gr}_{a}\left(R^{\tilde{\nabla}^{\prime}}-R^{\tilde{\nabla}}\right)$ lies in the kernel of $\operatorname{gr} \partial_{V}^{*}$. By Lemma 2.3, we have

$$
\operatorname{gr}_{a}\left(R^{\tilde{\nabla}^{\prime}}-R^{\tilde{\nabla}}\right)=\left(\operatorname{gr} \partial_{V}\right)\left(\operatorname{gr}_{a}\left(\Phi^{\prime}-\Phi\right)\right)
$$

But Ker gr $\partial_{V}^{*} \cap \operatorname{Im} \operatorname{gr} \partial_{V}$ is trivial, hence $\operatorname{gr}_{a}\left(R^{\tilde{\nabla}^{\prime}}-R^{\tilde{\nabla}}\right)=0$. Thus $\operatorname{gr}_{a}\left(\Phi^{\prime}-\Phi\right)$ lies in the kernel of $\operatorname{gr} \partial_{V}$, and also in the image of $\operatorname{gr} \partial_{V}^{*}$, by assumption. Hence $\operatorname{gr}_{a}\left(\Phi^{\prime}-\Phi\right)=0$, and thus

$$
\tilde{\nabla}^{\prime}-\tilde{\nabla}^{\prime}=\Phi^{\prime}-\Phi \in \mathcal{E}^{1}(\text { End } V)^{a+1} .
$$

Remark. The construction of $\nabla$ as outlined above depends at first on some choices (e.g., the choice of the preimage $\Phi$ of $\varphi$ ). However, the uniqueness of the prolongation covariant derivative shows that the result of the construction is independent of all choices. Hence the prolongation covariant derivative is invariant-it only depends on the data of the chosen parabolic structure and the bundle $V$. 


\section{Prolongation of the first BGG operator}

The BGG complexes are sequences of invariant differential operators on a homogeneous model for a given parabolic geometry. A curved version of them, i.e., an extension of operators in the sequence to invariant differential operators on general (nonflat) manifolds with a given parabolic structure, was first constructed in [9] and the construction was simplified and extended in [5]. The first operator in such a sequence always gives an overdetermined system of invariant differential equations. A prolongation of this operator for the case of 1-graded parabolic geometries was constructed in [4]. However, the methods used there needed a choice of a Weyl structure, hence the resulting covariant derivative was not invariant. We are now going to show that the normalization of tractor covariant derivatives described in this paper can be used to obtain invariant (natural) prolongations.

We begin by introducing the setting and basic operators of the BGG machinery in a generalized version needed for the next section. Let $V$ be a tractor bundle over $M$ with a covariant derivative $\nabla$ and the exterior covariant derivative $d^{\nabla}: \mathcal{E}^{k}(V) \rightarrow \mathcal{E}^{k+1}(V)$. Recall from the above that we have a well defined differential $\partial^{*}=\partial_{V}^{*}: \mathcal{E}^{k+1}(V) \rightarrow \mathcal{E}^{k}(V)$. The property $\partial^{*} \circ \partial^{*}=0$ allows us to define the cohomology $H_{k}$ as the vector bundle quotient $H_{k}=\operatorname{Ker} \partial^{*} / \operatorname{Im} \partial^{*}$, where $\operatorname{Ker} \partial^{*} \subset \mathcal{E}^{k}(V)$ is the space of cycles and $\operatorname{Im} \partial^{*} \subset \mathcal{E}^{k}(V)$ is the space of boundaries. The canonical surjection $\operatorname{Ker} \partial_{V}^{*} \subset \mathcal{E}^{k}(V)$ $\rightarrow H_{k}$ will be denoted by $\Pi_{k}$.

Due to regularity of the parabolic geometry under consideration, the operators $d^{\nabla}$ are homogeneous of degree zero with respect to the natural filtration of the spaces $\mathcal{E}^{k}(V)$ and they induce the algebraic differential gr $\partial_{V}: \operatorname{gr}\left(\mathcal{E}^{k}(V)\right) \rightarrow \operatorname{gr}\left(\mathcal{E}^{k+1}(V)\right)$ on the associated graded spaces. Thus it is possible to regard $d^{\nabla}$ as a natural lift of gr $\partial_{V}$ to a differential operator from $\mathcal{E}^{k}(V)$ to $\mathcal{E}^{k+1}(V)$.

The main ingredients in the BGG machinery are the differential splitting operators $L_{k}: H_{k} \rightarrow \operatorname{Ker} \partial_{V}^{*} \subset \mathcal{E}^{k}(V)$ with the property $\partial^{*} \circ d^{\nabla} \circ L_{k}=0$. This allows one to define the BGG operators $D_{k}: H_{k} \rightarrow H_{k+1}$ in the obvious way: $D_{k}:=\Pi_{k} \circ d^{\nabla} \circ L_{k}$. The definition is encoded in the diagram

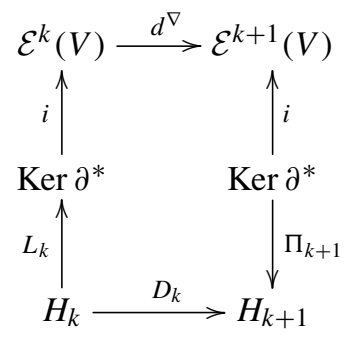

where $i$ denotes inclusion.

We shall introduce the construction of the splitting operators in a more general situation, where the exterior covariant derivatives $d^{\nabla}$ on $\mathcal{E}^{k}(V)$ will be replaced by general differential operators $E_{k}$ with suitable properties (see the theorem below). The operators $D_{k}$ are defined by the same construction as the BGG operators and they depend, in 
general, on the choice of $E_{k}$. The theorem below shows that for certain classes of operators $E_{k}$, the resulting operators $L_{k}$ and $D_{k}$ do not change.

Theorem 3.1. Let $\left(\mathcal{E}^{k}(V)\right)^{j}$ denote the filtration on $\mathcal{E}^{k}(V)$ and let $\operatorname{gr}\left(\mathcal{E}^{k}(V)\right)$ denote the associated graded bundle, and similarly for $\mathcal{E}^{k+1}(V)$. Let $E_{k}$ be a filtration preserving differential operator from $\mathcal{E}^{k}(V)$ to $\mathcal{E}^{k+1}(V)$ with the property that the associated graded map coincides with $\mathrm{gr} \partial$. Then for every $\sigma \in H_{k}$, there exists a unique element $s \in \operatorname{Ker} \partial^{*}$ with the following properties:

(1) $\Pi_{k}(s)=\sigma$,

(2) $E_{k}(s) \in \operatorname{Ker} \partial^{*}$.

Moreover, the mapping $L_{k}$ defined by $\sigma \mapsto L_{k}(\sigma):=s$ is given by a differential operator. The corresponding operator $D_{k}$ is then defined by

$$
D_{k}:=\Pi_{k+1} \circ E_{k} \circ L_{k}: H_{k} \rightarrow H_{k+1}
$$

Suppose that we change the operator $E_{k}$ to $\tilde{E}_{k}=E_{k}+\Phi_{k}$, where $\Phi_{k}: \mathcal{E}_{k}(V) \rightarrow$ $\mathcal{E}_{k+1}(V)$ is a differential operator with values in $\operatorname{Im} \partial^{*}$ and preserving the filtration, with the property that the associated graded map is trivial. Then the construction does not change the splitting operator $L_{k}$ and the operator $D_{k}$.

Proof. The first part of the proof follows the standard line of argument. The operator $\partial^{*} \circ E_{k}$ acts on $\mathcal{E}^{k}(V)$ and it preserves $\operatorname{Im} \partial^{*}$. It preserves the filtration and its graded version is, by assumption, given by $\operatorname{gr}\left(\partial^{*}\right) \circ \operatorname{gr}(\partial)$, which is invertible on $\operatorname{Im} \partial^{*}$. Hence also $\partial^{*} \circ E_{k}$ is invertible on $\operatorname{Im} \partial^{*}$ and it is possible to show that its inverse $Q$ is a differential operator.

We can then define a differential operator $\hat{L}_{k}:=\mathrm{Id}-Q \circ \partial^{*} \circ E_{k}$, which restricts to zero on $\operatorname{Im} \partial^{*}$. Hence it induces a well-defined differential operator $L_{k}$ from $H_{k}$ to Ker $\partial^{*} \subset \mathcal{E}^{k}(V)$. It is easy to check that the operator $L_{k}$ has the properties

$$
\operatorname{Im} L_{k} \subset \operatorname{Ker} \partial^{*}, \quad \Pi_{k} \circ L_{k}=\mathrm{Id}, \quad \partial^{*} \circ E_{k} \circ L_{k}=0 .
$$

To show that $L_{k}$ is uniquely characterized by these properties, let us consider $s_{1}, s_{2} \in$ $\operatorname{Ker} \partial^{*}$ such that $E_{k}\left(s_{i}\right) \in \operatorname{Ker} \partial^{*}, i=1,2$, and $\Pi_{k}\left(s_{1}\right)=\Pi_{k}\left(s_{2}\right)$. Then the difference $s=s_{1}-s_{2}$ belongs to $\operatorname{Im} \partial^{*}$. By definition of $\hat{L}_{k}$, the relation $\partial^{*} \circ E_{k}(s)=0$ implies $\hat{L}_{k}(s)=s$. On the other hand, $\hat{L}_{k}$ is trivial on $\operatorname{Im} \partial^{*}$. Hence $\hat{L}_{k}(s)=0$.

To prove the last statement of the theorem, we shall consider a section $s$ of $\mathcal{E}^{k}(V)$. The new operator $\tilde{E}_{k}$ preserves the filtration and the induced graded map is still gr $\partial$. Since $\left(\tilde{E}_{k}-E_{k}\right) s$ belongs to $\operatorname{Im} \partial_{V}^{*}$, one has $\tilde{E}_{k}(s) \in \operatorname{Ker} \partial_{V}^{*}$ iff $E_{k}(s) \in \operatorname{Ker} \partial_{V}^{*}$, which shows that $\tilde{L}_{k}=L_{k}$. Thus, for $\sigma \in H_{k}$, one has $\left(\tilde{E}_{k} \tilde{L}_{k}-E_{k} L_{k}\right) \sigma \in \operatorname{Im} \partial_{V}^{*}$, but this lies in the kernel of the projection $\Pi_{k+1}: \operatorname{Ker} \partial^{*} \rightarrow H_{k+1}$.

Now we want to discuss the relation between $\operatorname{Ker} E_{k}$ and $\operatorname{Ker} D_{k}$. For that, we have to consider two consecutive operators $E_{k}$ and $E_{k+1}$ at the same time. They define two split- 
ting operators $L_{k}$ and $L_{k+1}$. We get in this way the diagram

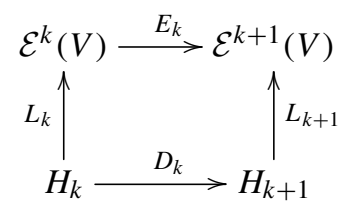

which, in general, does not commute but there is a convenient criterion for its commutativity.

Theorem 3.2. The diagram (4) commutes if and only if $\partial^{*} \circ E_{k+1} \circ E_{k}(s)=0$ for all sections $s \in \operatorname{Im} L_{k} \subset \mathcal{E}^{k}(V)$.

Proof. The values of $L_{k}$ are uniquely characterized by the conditions $L_{k}(\sigma) \in \operatorname{Ker} \partial^{*}$ and $E_{k} \circ L_{k}(\sigma) \in \operatorname{Ker} \partial^{*}$. Similarly, the values of $L_{k+1}$ are characterized by $L_{k+1}(\tau) \in \operatorname{Ker} \partial^{*}$ and $E_{k+1} \circ L_{k+1}(\tau) \in \operatorname{Ker} \partial^{*}$. Hence $E_{k} \circ L_{k}(\sigma)=L_{k+1} \circ D_{k}(\sigma)$ iff $E_{k+1} \circ E_{k} \circ L_{k}(\sigma) \in$ Ker $\partial^{*}$ for all $\sigma \in H_{k}$.

If the diagram above is commutative, we immediately get a one-to-one correspondence between $\operatorname{Ker} E_{k} \cap \operatorname{Ker} \partial^{*}$ and $\operatorname{Ker} D_{k}$.

Theorem 3.3. Suppose that the diagram (4) commutes. Then $\Pi_{k}$ and $L_{k}$ restrict to inverse isomorphisms between $\operatorname{Ker} E_{k} \cap \operatorname{Ker} \partial^{*}$ and $\operatorname{Ker} D_{k}$.

Proof. Let $s$ be in Ker $E_{k} \cap \operatorname{Ker} \partial^{*}$. Then $s=L_{k}\left(\Pi_{k}(s)\right)$ by definition of $L_{k}$, and $\Pi_{k}(s) \in$ Ker $D_{k}$ by definition of $D_{k}$.

On the other hand, if $D_{k}(\sigma)=0$, then commutativity of the diagram implies that also

$$
L_{k+1} \circ D_{k}(\sigma)=E_{k} \circ L_{k}(\sigma)=0,
$$

hence $L_{k}(\sigma) \in \operatorname{Ker} E_{k} \cap \operatorname{Ker} \partial^{*}$.

And by definition of $L_{k}$, we have $\Pi_{k} \circ L_{k}=\mathrm{Id}$.

Now we can return to the properties of the prolongation covariant derivative $\nabla$ on $V$. Using the above claims in the special case of the first square and the operators $E_{0}=\nabla$ and $E_{1}=d^{\nabla}$, we see immediately that $E_{1} \circ E_{0}=R^{\nabla}$. Hence we get the following corollary.

Corollary 3.1. Consider a tractor bundle $V$ and the corresponding prolongation covariant derivative $\nabla$. Set $E_{0}=\nabla$ and $E_{1}=d^{\nabla}$. Then the square constructed using these two operators commutes and the covariant derivative $\nabla$ gives a prolongation of the first $B G G$ operator $D_{0}$. In particular, the splitting operator $L_{0}$ induces a one-to-one correspondence between the space of parallel sections of $V$ with respect to $\nabla$ and the kernel of the first $B G G$ operator $D_{0}$.

Remark. In the case of a 1-graded geometry, it was shown in [4] that the map $L_{0}$ : $H_{0} \rightarrow V$ induces an isomorphism of $J^{k} H_{0}$ with $V_{\leq k}$ for every $k$ such that the homology of $H_{1}\left(\mathfrak{g}_{-}, \mathbb{V}\right)$ sits in homogeneity $>k$. Thus, for every operator $\tilde{D}_{0}: H_{0} \rightarrow H_{1}$ which differs from the standard BGG operator $D_{0}$ by a linear differential operator of 
order $\leq k$, there is a map $\Psi \in \mathcal{E}^{1}($ End $V)$ with values in $\operatorname{Ker} \partial_{V}^{*}$ whose induced first BGG operator coincides with $\tilde{D}_{0}$. The mapping $\Psi$ is unique up to maps in $\mathcal{E}^{1}$ (End $V$ ) with values in $\operatorname{Im} \partial_{V}^{*}$, and it is thus easy to see that the resulting normalized connection $\tilde{\nabla}=\nabla+\Psi+\Phi$ does not depend on the choice of $\Psi$. Thus, natural deformations of $D_{0}$ of low enough order can be prolonged naturally as well. We remark that a similar procedure works in the case of general graded parabolic geometries, where one has to use the filtration of the manifold for a suitable version of jet bundles (cf. [27, 28]).

\section{Prolongation covariant derivatives for the whole BGG sequence}

In this section we shall treat the problem considered above in the case of other squares of the BGG sequence. We want to deform the exterior covariant derivative $d^{\nabla}$ on $k$ forms in such a way that all squares in the generalized BGG construction will commute, and, at the same time, the BGG operators $D_{k}$ will not change. In fact, we shall succeed in keeping both the BGG operators $D_{k}$ and the splitting operators $L_{k}$ unchanged. The deformation of $d^{\nabla}$ on $\mathcal{E}^{k}(V)$ will have, however, a different character. It will be of the form $E_{k}:=d^{\nabla}+\Phi_{k}$, where $\Phi_{k}$ is a linear differential operator mapping $\mathcal{E}^{k}(V)$ to $\mathcal{E}^{k+1}(V)$. Hence the deformation $\Phi_{k}$ will not, in general, be algebraic. Necessary tools were already prepared in the previous section (Theorems 3.1-3.3). Methods described in this section can also be applied to the first square but they give a different answer (and also in this case the deformation $\Phi_{0}$ will not be algebraic in general).

To describe allowed deformations of the exterior derivative $d^{\nabla}$, we shall introduce the following notation. There are two different filtrations on the space $A:=$ $\operatorname{Hom}\left(\mathcal{E}^{k}(V), \mathcal{E}^{k+1}(V)\right)$. The diagonal filtration $A^{j}$ is induced by the standard filtration on $\mathcal{E}^{k}(V)$, which is defined by the condition $\Phi(s) \in \mathcal{E}^{k+1}(V)^{a+j}$ for all $s \in \mathcal{E}^{k}(V)^{a}$. The other (vertical) filtration $A^{a}$ is defined by the condition $\Phi(s) \in \mathcal{E}^{k+1}(V)^{a}$ for all $s \in \mathcal{E}^{k}(V)$. In this section, we shall use the symbols $\partial$ and $\partial^{*}$ for the Kostant differentials associated to the spaces $\mathcal{E}^{k}(V)$. Recall that the class $\mathcal{C}$ of admissible covariant derivatives on $V$ was defined by

$$
\mathcal{C}=\left\{\nabla=\nabla^{\omega}+\Phi \mid \Phi \in \operatorname{Im}\left(\partial_{V}^{*} \otimes \operatorname{Id}_{V^{*}}\right), \Phi \in \mathcal{E}^{1}(\text { End } V)^{1}\right\} .
$$

We shall consider the following spaces $\mathcal{C}_{k}$ of deformations.

Definition 4.1. The space of allowed deformations will be defined by

$$
\mathcal{C}_{k}:=\left\{E_{k} \in \operatorname{Hom}\left(\mathcal{E}^{k}(V), \mathcal{E}^{k+1}(V)\right) \mid E_{k}=d^{\nabla}+\Phi, \Phi \in A^{1}, \operatorname{Im} \Phi \subset \operatorname{Im} \partial^{*}\right\} .
$$

Theorem 4.2. (1) Let $\nabla$ be any covariant derivative from $\mathcal{C}$. Let us consider the $B G G$ sequence with the splitting operators $L_{k}$ and the $B G G$ operators $D_{k}$ induced (via Theorem 3.1) by the operators $E_{k}=d^{\nabla}$,

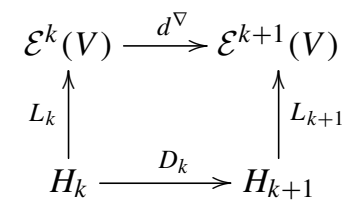


Then there exists a collection of differential operators $\Phi_{k} \in \mathcal{C}_{k}$ such that $\partial^{*} \circ d^{\nabla} \circ$ $\left(d^{\nabla}+\Phi_{k}\right)=0$. Moreover, the collection $\Phi_{k}$ with these properties is unique.

(2) As a consequence, the diagrams

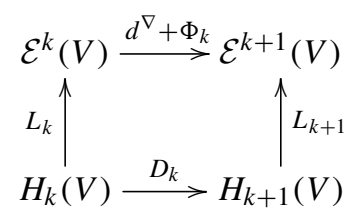

commute for all $k=0,1, \ldots, n-1$. Moreover, if $\nabla$ depends only on data of the chosen parabolic geometry, the same is true for the operators $E_{k}=d^{\nabla}+\Phi_{k}$.

Proof. Let us choose $k=0, \ldots, n-1$ and consider the square (6) in the generalized BGG sequence constructed using operators $d^{\nabla}$, where $\nabla$ is any covariant derivative from $\mathcal{C}$. We shall first prove the first assertion of the theorem.

The spaces $\left\{A^{1} \cap A^{a}\right\}_{a=0}^{r}$ form a decreasing filtration of the space $A^{1}$ with $a=$ $0, \ldots, r$. The filtration is preserved by the maps $\partial_{V}$ and $\partial_{V}^{*}$, hence they induce maps on the associated graded bundle (we denote them for simplicity by the same symbols as for the full filtration of $A$ ). We can consider the Kostant Laplacian $\square=\operatorname{gr} \partial_{V}^{*}$ gr $\partial_{V}+$ $\operatorname{gr} \partial_{V} \operatorname{gr} \partial_{V}^{*}$. The standard Kostant decomposition says that Ker $\square, \operatorname{Im} \operatorname{gr} \partial_{V}^{*}$ and $\operatorname{Im} \operatorname{gr} \partial_{V}$ are complementary subspaces of the graded bundle $\operatorname{gr} \mathcal{E}^{i}(V)^{1}$. In particular, $\square$ is invertible on $\operatorname{Im}$ gr $\partial_{V}^{*}$.

Let us consider two consecutive squares with operators $E_{k}=d^{\nabla}$ and $E_{k+1}=d^{\nabla}$. We know that the operator $G:=\partial^{*} \circ E_{k+1} \circ E_{k}$ belongs to $A^{1}$ and that the $k$-th square is commutative iff $G=0$. If it is not the case, we shall consider the maximal index $a=0$ with the property that $G \in A^{a}$.

The map $\Phi^{(1)}=-\square^{-1} \operatorname{gr}(G)$ can be lifted to a linear algebraic map $\Phi^{(1)}: \mathcal{E}^{k}(V) \rightarrow$ $\mathcal{E}^{k+1}(V)$ (e.g., by choosing a Weyl structure) and we shall define the first iteration $E_{k}^{(1)}=$ $d^{\nabla}+\Phi^{(1)}$. Note that the lowest homogeneous component of $E_{k}^{(1)}$ remains to be $\partial_{V}$ and that the image of $E_{k}^{(1)}$ is a subset of $\operatorname{Im} \partial^{*}$.

Since

$$
E_{k+1} \circ E_{k}^{(1)}-E_{k+1} \circ E_{k}=d^{\nabla} \circ \Phi^{(1)},
$$

we get

$$
\begin{aligned}
\left.\operatorname{gr}_{a}\left(\partial^{*} \circ E_{k+1} \circ E_{k}^{(1)}\right)\right) & =\operatorname{gr}_{a}\left(G+\partial^{*} \circ d^{\nabla} \circ \Phi^{(1)}\right) \\
& =\operatorname{gr}_{a}(G)-\left(\operatorname{gr} \partial^{*}\right)\left(\operatorname{gr} \partial_{V}\right)\left(\square^{-1}\left(\operatorname{gr}_{a}(G)\right)\right)=0 .
\end{aligned}
$$

Hence the first order differential operator $G^{(1)}:=\partial^{*} \circ E_{k+1} \circ E_{k}^{(1)}$ belongs to $A^{a+1}$.

The same procedure will be repeated inductively. If we define

$$
\Phi^{(2)}=-\left(\operatorname{gr} \partial^{*}\right) \square^{-1} \operatorname{gr}_{a+1}\left(G^{(1)}\right)
$$


we can again lift this first order differential operator to a first order differential operator $\Phi^{(2)}: \mathcal{E}^{k}(V) \rightarrow \mathcal{E}^{k+1}(V)$ and we can define the next iteration by

$$
E_{k}^{(2)}:=E_{k}^{(1)}+\Phi^{(2)} .
$$

Then we get

$$
\begin{aligned}
\left.\operatorname{gr}_{a}\left(\partial_{V}^{*} \circ d^{\nabla} \circ E_{k}^{(2)}\right)\right) & =\operatorname{gr}_{a}\left(G^{(1)}+\partial_{V}^{*} \circ d^{\nabla} \circ \Phi^{(2)}\right) \\
& =\operatorname{gr}_{a}\left(G^{(1)}\right)-\left(\operatorname{gr} \partial_{V}^{*}\right)\left(\operatorname{gr} \partial_{V}\right)\left(\square^{-1}\left(\operatorname{gr}_{a}\left(G^{(1)}\right)\right)\right)=0 .
\end{aligned}
$$

Hence the first order differential operator $G^{(2)}:=\partial_{V}^{*} \circ d^{\nabla} \circ E_{k}^{(2)}$ belongs to $A^{a+2}$.

It is clear that after a finite number of iterations, we shall get the existence part of the theorem.

The proof of the uniqueness part is similar to the procedure employed in Lemma 2.5. Suppose that we have two differential operators $\Phi_{k}^{\prime}$ and $\Phi_{k}^{\prime \prime}$ satisfying the conditions of the theorem. Their difference $\Phi=\Phi_{k}^{\prime}-\Phi_{k}^{\prime \prime}$ satisfies $\partial_{V}^{*}\left(d^{\nabla} \circ \Phi\right)=0$. To show that $\Phi=0$, suppose that $\Phi$ is nontrivial and consider the largest $a$ such that $\Phi^{a}$ is nontrivial. Then we know that $\operatorname{gr}_{a}\left(d^{\nabla} \circ \Phi\right)=\left(\operatorname{gr}_{V}\right)\left(\operatorname{gr}_{a} \Phi\right)$, hence $\left(\operatorname{gr}_{V} \partial_{V}\right)\left(\operatorname{gr}_{a} \Phi\right)$ is at the same time in $\operatorname{Im} \operatorname{gr} \partial_{V}$ and $\operatorname{Ker} g r \partial_{V}^{*}$, so it is 0 . By definition, $\operatorname{gr}_{a} \Phi$ also belongs to $\operatorname{Im} \partial_{V}^{*}$, hence $\operatorname{gr}_{a} \Phi$ is trivial and we have a contradiction.

As for the second part of the theorem, let us consider two consecutive squares in the BGG construction induced by $E_{k}=d^{\nabla}$, containing the operators $D_{k}$ and $D_{k+1}$. If $\Phi_{k}$ is the deformation constructed above, then the replacement of $E_{k}=d^{\nabla}$ by $\tilde{E}_{k}=$ $d^{\nabla}+\Phi_{k}$ leads to the same splitting operator $L_{k}$. Hence by the first part of the theorem, the $k$-th diagram commutes. Note that changing the next operator $E_{k+1}$ will not change the splitting operator $L_{k+1}$, hence the commutativity of the $k$-th diagram is preserved.

Finally, during the construction there were several choices made but due to the uniqueness of the result, the construction depends only on the data of the chosen parabolic geometry. The same is true for the covariant derivative $\nabla$.

\section{Examples}

In this section we want to illustrate the general results presented above by explicit examples showing the form of the prolongation covariant derivative in some simple situations. A more comprehensive set of examples is given in [24].

To calculate the prolongation covariant derivative of the first BGG operator $D_{0}$ for some tractor bundle $V=\mathcal{G} \times{ }_{P} \mathbb{V}$, we employ the theory of Weyl structures [7], [8]. All of our examples below will be $|1|$-graded parabolic geometries, $\mathfrak{g}=\mathfrak{g}_{-1} \oplus \mathfrak{g}_{0} \oplus \mathfrak{g}_{1}$. Modding out $P_{+} \cong \mathfrak{g}_{1}$ of the parabolic structure bundle $\mathcal{G}$, one obtains $\mathcal{G}_{0}:=\mathcal{G} / P_{+}$, which is a $G_{0}$-principal bundle over $M$. A splitting $\sigma: \mathcal{G}_{0} \rightarrow \mathcal{G}$ of the canonical projection $\mathcal{G} \rightarrow \mathcal{G}_{0}$ is called a Weyl structure, and for our geometric structures below this can be identified with the choice of a Weyl connection, which is a linear connection $D$ compatible with the geometry. Under such a choice, all $P$-associated bundles reduce to $G_{0}$-associated bundles, and in particular one gets a decomposition of every tractor bundle $V$ which depends on 
the choice of the Weyl structure [8]. In particular, the adjoint tractor bundle $\mathcal{A} M=\mathcal{G} \times{ }_{P} \mathfrak{g}$ decomposes into $\mathcal{A}_{-} M \oplus \mathcal{A}_{0} M \oplus \mathcal{A}_{1} M$, with $\mathcal{A}_{-} M \cong T M$ and $A_{+} M \cong T^{*} M$. The Lie algebraic action of $\mathfrak{g}$ on $\mathbb{V}$ gives rise to an action $\bullet$ of $\mathcal{A} M$ on $V$, which we can restrict to $T M$ and $T^{*} M$. The tractor covariant derivative $\nabla^{\omega}$ can be written as $\nabla^{\omega}=\partial+D+\mathrm{P} \bullet$ : the map $\partial: V \rightarrow \Omega^{1}(M, V)$ is obtained by the action of $T M \hookrightarrow \mathcal{A} M$ on $V$, and $\mathrm{P} \bullet: V \rightarrow \Omega^{1}(M, V)$ is induced by the action of the second slot of the (generalized) Schouten tensor $\mathrm{P} \in \mathcal{E}_{a b}$ of $D$, which will be symmetric for our choices of $D$. Recall that this decomposition of $\nabla^{\omega}$ depends on the choice of the Weyl structure $\sigma: \mathcal{G}_{0} \rightarrow \mathcal{G}$ resp. Weyl connection $D$.

In our explicit formulas, we employ abstract index notation [29]: $\mathcal{E}_{a}=\Omega^{1}(M)$, $\mathcal{E}^{a}=\mathfrak{X}(M)$, and multiple indices indicate tensor products. Round brackets denote symmetrizations of the indices enclosed, and square brackets denote skew symmetrizations. A subscript zero indicates taking the trace-free part.

We now prolong an interesting equation in projective geometry which has already been treated in [17] by different methods. Next we consider three well known overdetermined equations in conformal geometry, which govern Einstein rescalings, conformal Killing forms and twistor spinors. Finally we analyse an equation for Grassmannian structures of type $(2, q), q>2$. For a more detailed exposition of explicit calculations $\mathrm{cf}$. [22]-[24].

\subsection{An example in projective geometry}

Let $M$ be an orientable manifold of dimension $n$ endowed with a projective class of linear, torsion-free connections [D]; here $D$ and $D^{\prime}$ are projectively equivalent if there is a $\Upsilon \in \mathcal{E}^{1}$ such that

$$
D_{a}^{\prime} \omega_{b}=D_{a} \omega-\Upsilon_{a} \omega_{b}-\Upsilon_{b} \omega_{a}
$$

(see e.g. [14]). For simplicity, we will assume that our chosen representatives $D \in[D]$ preserve a volume form on $T M$.

To define projectively invariant operators we need to employ densities, which are sections of line bundles $\mathcal{E}[w], w \in \mathbb{R}$, associated to the full GL(n)-frame bundle of $T M$ via the 1-dimensional representation

$$
C \in \mathrm{GL}(n) \mapsto|\operatorname{det} C|^{w(n+1) / n} \in \mathbb{R}_{+} .
$$

With this parametrization, sections of the bundles $\mathcal{E}[w]$ are often called projective densities. Assume $n \geq 2$. We are going to prolong the following projectively invariant operator, which is written down with respect to a $D \in[D]$, but does not depend on this choice:

$$
D_{0}: \mathcal{E}^{(a b)}[-2] \rightarrow \mathcal{E}_{c}^{(a b)}{ }_{0}[-2], \quad \sigma^{a b} \mapsto D_{c} \sigma^{a b}-\frac{1}{n+1} \delta_{c}^{(a} D_{p} \sigma^{b) p} .
$$

$D_{0}$ projects the Levi-Civita derivative of a symmetric two-tensor $\sigma$ to its trace-free part. This operator was discussed in [17], where M. Eastwood and V. Matveev showed that this equation governs the metrizability of a projective class of covariant derivatives. 
5.1.1. The projective structure as a parabolic geometry. It is a classical result that $(M,[D])$ is equivalent to a unique Cartan geometry $(\Gamma, \omega)$ of type $(G, P)=$ $(\mathrm{SL}(n+1), P)$ with $P$ the stabilizer of a ray in $\mathbb{R}^{n+1}$ (cf. $\left.[11,31,8]\right)$.

The Lie algebra $\mathfrak{g}=\mathfrak{s l}(n+1)$ is 1-graded, $\mathfrak{g}=\mathfrak{g}_{-1} \oplus \mathfrak{g}_{0} \oplus \mathfrak{g}_{1}=\mathbb{R}^{n} \oplus \mathfrak{g l}(n) \oplus\left(\mathbb{R}^{n}\right)^{*}$, where an element $X \oplus(\alpha \mathrm{id}+A) \oplus \varphi \in \mathfrak{g}$ for $\alpha \in \mathbb{R}, A \in \mathfrak{s l}(n)$ corresponds to the matrix

$$
\left(\begin{array}{cc}
-\alpha \frac{n}{n+1} & -\varphi \\
X & \frac{1}{n+1} \alpha \mathbb{I}_{n}+A
\end{array}\right)
$$

The actions of $\mathfrak{g}_{0}=\mathfrak{g l}(n) \subset \mathfrak{g}$ on $\mathfrak{g}_{-1}=\mathbb{R}^{n}$ and $\mathfrak{g}_{1}=\left(\mathbb{R}^{n}\right)^{*}$ are the standard representation and its dual.

The curvature of the Cartan connection form $\omega$ can be regarded as an element of $\mathcal{E}^{2}(\mathcal{A} M)$, with $\mathcal{A} M=\Gamma \times{ }_{P} \mathfrak{g}$ the adjoint tractor bundle, and is written

$$
K=\left(\begin{array}{cc}
0 & -A_{a c_{1} c_{2}} \\
0 & C_{c_{1} c_{2}}{ }_{b}
\end{array}\right)
$$

with $A$ the Cotton-York tensor and $C$ the (projectively invariant) Weyl curvature (cf. [14]).

1 -forms and vector fields include into $\mathcal{A} M$ as

$$
\eta_{a} \in T^{*} M \mapsto\left(\begin{array}{cc}
0 & -\eta_{a} \\
0 & 0
\end{array}\right) \in \mathcal{A} M, \quad \xi^{a} \in T M \mapsto\left(\begin{array}{ll}
0 & 0 \\
\xi & 0
\end{array}\right) \in \mathcal{A} M .
$$

5.1.2. The operator $D_{0}$ as the first $B G G$ operator. Let $V:=\Gamma \times{ }_{P} S^{2} \mathbb{R}^{n+1}$. With respect to a choice of a Weyl connection $D \in[D]$, a section $s$ of $V$ can be written

$$
[s]_{D}=\left(\begin{array}{c}
\rho \\
\mu^{a} \\
\sigma^{a b}
\end{array}\right) \in\left(\begin{array}{c}
V_{2} \\
V_{1} \\
V_{0}
\end{array}\right):=\left(\begin{array}{c}
\mathcal{E}[-2] \\
\mathcal{E}^{a}[-2] \\
\mathcal{E}^{(a b)}[-2]
\end{array}\right)
$$

We will need that on the first chain spaces the Lie algebra differentials $\partial$ and $\partial^{*}$ are explicitly given by

$$
\begin{aligned}
& \partial\left(\begin{array}{c}
\rho \\
\mu^{a} \\
\sigma^{a b} .
\end{array}\right)=\left(\begin{array}{c}
0 \\
\rho \delta_{c}^{a} \\
\delta_{c}\left(a \mu^{b)}\right.
\end{array}\right), \quad \partial\left(\begin{array}{c}
\rho_{c} \\
\mu_{c}^{a} \\
\sigma_{c}^{a b} .
\end{array}\right)=\left(\begin{array}{c}
0 \\
2 \delta_{\left[c_{1}\right.}{ }^{a b} \rho_{\left.c_{2}\right]} \\
2 \delta_{\left[c_{1}\right.}\left(a_{1} \mu_{\left.c_{2}\right]}{ }^{a}\right)
\end{array}\right), \\
& \partial^{*}\left(\begin{array}{c}
\rho_{c} \\
\mu_{c}^{a} \\
\sigma_{c}{ }^{a b} .
\end{array}\right)=\left(\begin{array}{c}
-2 \mu_{p}{ }^{p} \\
-2 \sigma_{p}{ }^{p a} \\
0
\end{array}\right), \quad \partial^{*}\left(\begin{array}{c}
\rho_{c_{1} c_{2}}{ } \\
\mu_{c_{1} c_{2}}{ }^{a} \\
\sigma_{c_{1} c_{2}}{ }^{2} .
\end{array}\right)=\left(\begin{array}{c}
2 \mu_{c p}{ }^{p} \\
2 \sigma_{c p} p a \\
0
\end{array}\right) .
\end{aligned}
$$

As bundles with structure group $G_{0}, V_{2}, V_{1}$ and $T^{*} M \otimes V_{2}$ are irreducible and are contained in the image of $\partial^{*} ; T^{*} M \otimes V_{1}$ decomposes into the trace-free part $\operatorname{Im} \partial^{*} \cap T^{*} M \otimes V_{1}$ and the trace part, which lies in the image of $\partial$. The Kostant Laplacian $\square$ acts by

$$
\square\left(\begin{array}{c}
\rho_{c_{1} c_{2}} \\
\mu_{c_{1} c_{2}} a \\
\sigma_{c_{1} c_{2}} a b
\end{array}\right)=\left(\begin{array}{c}
-2 n \rho_{c_{1} c_{2}} \\
-(n+1) \mu_{c_{1} c_{2}}{ }^{a} \\
0
\end{array}\right)
$$


on $V$, by multiplication with $-2(n-1)$ on $T^{*} M \otimes V_{2}$ and by multiplication with $-n$ on the trace-free part of $T^{*} M \otimes V_{1}$. This is all the algebraic information we need to calculate the splitting operators and the prolongation.

The tractor covariant derivative $\nabla^{\omega}$ on $V$ is easily calculated with the above actions of $\mathcal{E}_{a}$ and $\mathcal{E}^{a}$ on $V$ together with the formula $\nabla^{\omega}=\partial+D+\mathrm{P} \bullet$ :

$$
\nabla^{\omega}\left(\begin{array}{c}
\rho \\
\mu^{a} \\
\sigma^{a b}
\end{array}\right)=\left(\begin{array}{c}
D_{c} \rho-2 \mathrm{P}_{c a} \mu^{a} \\
D_{c} \mu^{a}-2 \mathrm{P}_{c b} \sigma^{a b}+\rho \delta_{c}{ }^{a} \\
D_{c} \sigma^{a b}+\delta_{c}^{(a} \mu^{b)}
\end{array}\right) .
$$

One calculates that the first splitting operator $L_{0}: \Gamma\left(H_{0}\right) \rightarrow \Gamma(V)$ is given by

$$
\sigma^{(a b)} \mapsto\left(\begin{array}{c}
\frac{1}{n(n+1)} D_{p} D_{q} \sigma^{p q}+\frac{1}{2 n} \mathrm{P}_{p q} \sigma^{p q} \\
-\frac{1}{n+1} D_{p} \sigma^{p a} \\
\sigma^{a b}
\end{array}\right)
$$

and the composition of $\nabla^{\omega} \circ L_{0}$ with the projection to the lowest slot is seen to yield the operator $D_{0}$ of (7).

5.1.3. Prolongation of $D_{0}$. We calculate the action of the curvature $K \in \Omega^{2}(M, \mathcal{A M})$ :

$$
K_{c_{1} c_{2}} \bullet\left(\begin{array}{c}
0 \\
0 \\
\sigma^{a b}
\end{array}\right)=\left(\begin{array}{c}
-2 A_{p c_{1} c_{2}} \mu^{p} \\
-2 A_{p c_{1} c_{2}} \sigma^{p a}+C_{c_{1} c_{2}}^{a}{ }_{p} \mu^{p} \\
2 C_{c_{1} c_{2}}{ }^{\left(a_{1}\right.} \sigma^{\left.a_{2}\right) p}
\end{array}\right)
$$

Therefore we define

$$
\Phi_{1}\left(\left(\begin{array}{c}
0 \\
0 \\
\sigma^{a b}
\end{array}\right)\right):=\left(\begin{array}{c}
0 \\
\bar{\Phi}_{1} \sigma \\
0
\end{array}\right):=-\square^{-1}\left(\partial^{*}\left(K \bullet\left(\begin{array}{c}
0 \\
0 \\
\sigma^{a b}
\end{array}\right)\right)\right)=\left(\begin{array}{c}
0 \\
\frac{2}{n} C_{c p}{ }^{a}{ }^{a} \sigma^{p q} \\
0
\end{array}\right) .
$$

Now the curvature of the modified connection $\nabla^{\omega}+\Phi_{1}$ is $R=K \bullet+d^{\nabla} \Phi_{1}$ since $\left(\Phi_{1} \wedge \Phi_{1}\right)(\xi, \eta)$ vanishes. For $\xi_{1}, \xi_{2} \in \mathfrak{X}(M)$ and $s \in V$,

$$
\begin{aligned}
\left(d^{\nabla} \Phi_{1}\right) s\left(\xi_{1}, \xi_{2}\right)= & \nabla_{\xi_{1}}\left(\Phi_{1}\left(\xi_{2}\right) s\right)-\Phi_{1}\left(\xi_{2}\right)\left(\nabla_{\xi_{1}} s\right)-\nabla_{\xi_{2}}\left(\Phi_{1}\left(\xi_{1}\right) s\right)+\Phi_{1}\left(\xi_{1}\right)\left(\nabla_{\xi_{2}} s\right) \\
& -\Phi_{1}\left(\left[\xi_{1}, \xi_{2}\right]\right) s .
\end{aligned}
$$

We may expand (10) and write $\left(d^{\nabla} \Phi_{1}\right) s$ as

$$
\left.\left(\begin{array}{c}
* \\
D_{\xi_{1}}\left(\bar{\Phi}_{1}\left(\xi_{2}\right) \sigma\right)-\bar{\Phi}_{1}\left(\xi_{2}\right)\left(D_{\xi_{1}} \sigma\right)-D_{\xi_{2}}\left(\bar{\Phi}_{1}\left(\xi_{1}\right) \sigma\right)+\bar{\Phi}_{1}\left(\xi_{1}\right)\left(D_{\xi_{2}} \sigma\right) \\
-\bar{\Phi}_{1}\left(\left[\xi_{1}, \xi_{2}\right]\right) \sigma \\
-\bar{\Phi}_{1}\left(\xi_{2}\right) \partial_{\xi_{1}} \varphi+\bar{\Phi}_{1}\left(\xi_{1}\right) \partial_{\xi_{2}} \varphi-\bar{\Phi}_{1}\left(\xi_{2}\right) \partial_{\xi_{1}} \mu+\bar{\Phi}_{1}\left(\xi_{1}\right) \partial_{\xi_{2}} \mu
\end{array}\right)\right)
$$

where we do not take care about the top component since it will vanish after an application of $\partial^{*}$. The lowest component is simply $\partial\left(\bar{\Phi}_{1} \sigma\right)=-\partial \square^{-1} \partial^{*}(K \bullet \sigma)$. Thus $\partial^{*}(R s)$ lies in the top slot (i.e., in homogeneity 1). So our first adjustment had the effect of moving the expression $\partial^{*}(R s)$ one slot up. 
The new connection $\nabla^{\omega}+\Phi_{1}$ has the following terms in the middle slot of the curvature $R_{\Phi_{1}}$ : From (11) we obtain the terms $2 D_{\left[c_{1}\right.} \bar{\Phi}_{\left.1 c_{2}\right]}$ and (via an application of the algebraic Bianchi identity for $C$ ), $C_{c_{1} c_{2} p} \mu^{p}$. By (9), the contribution of $K \bullet s$ to the middle slot is $-2 A_{p c_{1} c_{2}} \sigma^{p a}+C_{c_{1} c_{2} p}^{a} \mu^{p}$. In total, we find that the action of the curvature $R_{\Phi_{1}}$ is

$$
\left(\begin{array}{c}
\rho \\
\mu^{a} \\
\sigma^{a b}
\end{array}\right) \mapsto\left(\begin{array}{c}
* \\
\frac{2}{n}\left(D_{\left[c_{1}\right.} C_{\left.c_{2}\right] p}^{a}{ }_{q}\right) \sigma^{p q}-2 A_{p c_{1} c_{2}} \sigma^{p a}+2 C_{c_{1} c_{2}}^{a}{ }^{a} \mu^{p} \\
*
\end{array}\right) .
$$

The entries $(*)$ are irrelevant: the lowest slot is by construction already in the kernel of $\partial^{*}$ and the highest slot always lies in Ker $\partial^{*}$. Now define

$$
\Phi_{2}\left(\left(\begin{array}{c}
\rho \\
\mu^{a} \\
\sigma^{a b}
\end{array}\right)\right):=-\square^{-1} \partial^{*}\left(R_{\Phi_{1}}\left(\left(\begin{array}{c}
\rho \\
\mu^{a} \\
\sigma^{a b}
\end{array}\right)\right)\right)
$$

Using $D_{p} C_{c_{1} c_{2}}^{p}{ }_{a}=(n-2) A_{a c_{1} c_{2}}$ and trace-freeness of $C$, we calculate

$$
\Phi_{2}\left(\left(\begin{array}{c}
\rho \\
\mu^{a} \\
\sigma^{a b}
\end{array}\right)\right)=\left(\begin{array}{c}
-\frac{4}{n} A_{p c q} \sigma^{p q} \\
0 \\
0
\end{array}\right)
$$

and find that $\Phi:=\Phi_{1}+\Phi_{2} \in \Gamma\left(T^{*} M \otimes \operatorname{End}(V)\right)$ is

$$
\left(\begin{array}{c}
\rho \\
\mu^{a} \\
\sigma^{a b}
\end{array}\right) \mapsto \frac{2}{n}\left(\begin{array}{c}
-2 A_{p c q} \sigma^{p q} \\
C_{c p}^{a}{ }^{a} \sigma^{p q} \\
0
\end{array}\right)
$$

Now, with $R_{\Phi}$ the curvature of $\tilde{\nabla}=\nabla^{\omega}+\Phi$, one has by construction $\partial^{*} \circ R_{\Phi}=0$. Thus $\tilde{\nabla}$ is the prolongation covariant derivative for $\left(D_{c} \sigma^{a b}\right)_{0}=0$.

\subsection{Examples in conformal geometry}

Let $M$ be an $n$-manifold endowed with a conformal class [ $g$ ] of (pseudo-)Riemannian signature $(p, q)$ metrics. The conformal structure $(M,[g])$ is equivalent to a reduction of the structure group of the full frame bundle of $T M$ to a $\mathrm{CO}(p, q)=\mathbb{R}_{+} \times O(p, q)$-bundle $\mathcal{G}_{0} \rightarrow M$. To write down conformally invariant differential operators we will employ conformal density bundles $\mathcal{E}[w]$, which are associated to the 1-dimensional $\operatorname{CO}(p, q)$ representation $(\alpha, C) \in \operatorname{CO}(p, q) \mapsto \alpha^{w} \in \mathbb{R}_{+}$.

The conformal structure can be equivalently encoded as a parabolic geometry $(\mathcal{G}, \omega)$ of type $(\mathrm{SO}(p+1, q+1), P)$, with $P \subset \mathrm{SO}(p+1, q+1)$ the stabilizer of an isotropic ray in $\mathbb{R}^{p+1, q+1}$ (cf. $\left.[10,8]\right)$; the curvature of $\omega$ is an element $\kappa \in \mathcal{E}^{2}(\mathcal{A} M)$, with $\mathcal{A} M=$ $\mathcal{G} \times{ }_{P} \mathfrak{s o}(p+1, q+1)$, and has to satisfy the normalization condition $\partial^{*} \kappa=0$. Choosing a metric $g \in[g]$ yields its Levi-Civita connection $D$ on $T M$, which serves as a Weyl connection, and we make use of this to get explicit formulas for BGG operators in the following. 
Since the necessary explicit tractor calculations for the next three equations are available in [23], and in the case of the conformal Killing form equations rather long, we just summarize the important properties here and relate the results to our general theory.

5.2.1. Almost Einstein scales. Let now $\mathcal{T}:=\mathcal{G} \times{ }_{P} \mathbb{R}^{p+1, q+1}$ be the standard tractor bundle of the conformal structure, which is endowed with its normal tractor covariant derivative $\nabla^{\omega}$. It was already observed in [1] that parallel sections of $\nabla^{\omega}$ are in one-toone correspondence with solutions of $D_{0}$,

$$
D_{0}: \mathcal{E}[1] \rightarrow \mathcal{E}_{(a b)_{0}}[1], \quad \sigma \mapsto \mathbf{t f}\left(D_{a} D_{b} \sigma+\mathrm{P}_{a b} \sigma\right),
$$

where tf takes the trace-free part. This is a conformally invariant $2^{\text {nd }}$ order PDE, and its solutions $\sigma$ are Einstein rescalings or almost Einstein scales, [18]: $\sigma$ is nonvanishing on an open dense subset, and $\sigma^{-2} g$ is Einstein there. In particular, $\nabla^{\omega}$ is already the prolongation covariant derivative of this problem: it is also easy to see directly that its curvature $R^{\nabla^{\omega}}$ satisfies $\partial_{V}^{*}\left(R^{\nabla^{\omega}}\right)=0$ [23].

5.2.2. Conformal Killing forms. Let now $\mathcal{V}:=\Lambda^{k+1} \mathcal{T}$ be an exterior power of the conformal standard tractor bundle, which is again endowed with the normal tractor connection $\nabla^{\omega}$. An explicit tractor computation yields

$$
\begin{aligned}
D_{0}: \mathcal{E}_{\left[a_{1} \cdots a_{k}\right]}[k+1] & \rightarrow \mathcal{E}_{c\left[a_{1} \cdots a_{k}\right]}[k+1], \\
\sigma & \mapsto D_{c} \sigma_{a_{1} \cdots a_{k}}-D_{\left[a_{0}\right.} \sigma_{\left.a_{1} \cdots a_{k}\right]}-\frac{k}{n-k+1} g_{c\left[a_{1}\right.} g^{p q} D_{\mid p} \sigma_{\left.q \mid a_{2} \cdots a_{k}\right]},
\end{aligned}
$$

which is the projection of $D \sigma$ to the highest weight component in $\mathcal{E}_{c\left[a_{1} \cdots a_{k}\right]}[k+1]$. Solutions of $D_{0} \sigma=0$ are the conformal Killing forms on $M$. The equations governing conformal Killing forms were first prolonged by U. Semmelmann [30]. In [19] an invariant prolongation was calculated directly. The prolongation covariant derivative $\nabla$ for this equation is already fairly complicated to compute explicitly, and we refer to [23, 22] for this.

5.2.3. Twistor spinors. In the case where one has a reduction of the $\operatorname{CO}(p, q)$-bundle of $(M,[g])$ to a $\operatorname{CSpin}(p, q)=\mathbb{R}_{+} \times \operatorname{Spin}(p, q)$-bundle $\mathcal{G}_{0}$ one knows that $(M,[g])$ is a conformal spin structure. This structure is then equivalently described as a Cartan geometry of type (Spin $(p+1, q+1), P)$, with $P \subset \operatorname{Spin}(p+1, q+1)$ again the stabilizer of an isotropic ray in $\mathbb{R}^{p+1, q+1}$. Let $\Delta^{p+1, q+1}$ be the $\operatorname{Spin}(p+1, q+1)$ representation, which is decomposable in the case where $p+q$ is even. The corresponding associated tractor bundle is $\mathcal{S}=\mathcal{G} \times{ }_{P} \Delta^{p+1, q+1}$. Now let $\Delta^{p, q}$ be the spin representation of $\operatorname{Spin}(p, q)$, which we extend trivially to $\operatorname{CSpin}(p, q)$, and define $S:=\mathcal{G}_{0} \times{ }_{\operatorname{CSpin}(p, q)} \Delta^{p, q}$. Then the first BGG operator of $\mathcal{S}$ is

$$
D_{0}: \Gamma(S[1 / 2]) \rightarrow \Gamma\left(\mathcal{E}_{c} \otimes S[1 / 2]\right), \quad \sigma \rightarrow \operatorname{proj}\left(D_{c} \sigma\right) .
$$

This is the twistor operator: it is the composition of $D: \Gamma(S) \rightarrow \mathcal{E}_{c}(S)$ with proj, the projection to the kernel of Clifford multiplication. It is again well known [2, 3, 23] that solutions of $D_{0}$ are already in one-to-one correspondence with parallel sections of the normal tractor covariant derivative $\nabla^{\omega}$ on the spin tractor bundle $\mathcal{S}$. 


\subsection{An example in Grassmannian geometry}

Let $q \in \mathbb{N}, q>2$, and $M$ be an oriented $2 q$-dimensional manifold together with a rank 2 bundle $\mathcal{E}_{\alpha}$ and a rank $q$ bundle $\mathcal{E}^{\alpha^{\prime}}$. Assume there is an isomorphism of $T M$ with $\mathcal{E}_{\alpha} \otimes \mathcal{E}^{\beta^{\prime}}$, which will be fixed. We say that $M$ together with the identification $T M=\mathcal{E}_{\alpha}^{\beta^{\prime}}$ is a Grassmannian geometry of type $(2, q)$ if there exists a torsion-free linear connection $D$ on $T M$ which is the product of linear connections (again denoted by $D$ ) on $\mathcal{E}_{\alpha}$ and $\mathcal{E}^{\beta^{\prime}}$ (see [20], [8]). The class of all such connections are the Weyl connections of $\left(M, T M \cong \mathcal{E}_{\alpha}^{\beta^{\prime}}\right)$.

We are going to prolong the operator

$$
D_{0}: \mathcal{E}^{\left[\alpha^{\prime} \beta^{\prime}\right]} \rightarrow\left(\mathcal{E}_{\gamma^{\prime}}^{\gamma\left[\alpha^{\prime} \beta^{\prime}\right]}\right)_{0}, \quad u^{\alpha^{\prime} \beta^{\prime}} \mapsto D_{\gamma^{\prime}}^{\gamma} u^{\alpha^{\prime} \beta^{\prime}}+\frac{2}{1-q} \delta_{\gamma^{\prime}}^{\left[\alpha^{\prime}\right.} D_{\tau^{\prime}}^{\mid \gamma} u^{\left.\tau^{\prime} \mid \beta^{\prime}\right]}
$$

Thus, $D_{0}(u)$ is the projection of $D u$ to its trace-free part.

5.3.1. Grassmannian structures as parabolic geometries. Let $G=\operatorname{SL}(n), n=2+q$, and define $P$ as the stabilizer of a two-plane in $\left(\mathbb{R}^{n}\right)^{*}$. Regular, normal and torsion-free parabolic geometries $(\mathcal{G}, \omega)$ of type $(G, P)$ are Grassmannian structures. In the Cartan picture, $\mathcal{E}_{\alpha}$ and $\mathcal{E}^{\alpha^{\prime}}$ are associated to the $P$-representations $\left(\mathbb{R}^{p}\right)^{*}$, resp. $\mathbb{R}^{q}$.

Let $\mathcal{S}$ be the standard tractor bundle of $(\mathcal{G}, \omega)$, i.e., the associated bundle to the standard representation of $\operatorname{SL}(n)$. Via any Weyl structure $D, \mathcal{S}$ decomposes into $\mathcal{E}^{\alpha} \oplus \mathcal{E}^{\alpha^{\prime}}$.

The curvature $K \in \mathcal{E}^{2}(\mathcal{A} M)=\mathcal{E}^{2}(\mathcal{S})$ of the Cartan connection is of the form

$$
K=\left(\begin{array}{cc}
C_{c_{1} c_{2} \eta} & -A_{p c_{1} c_{2}} \\
0 & C^{\prime} \varphi^{\prime} \\
c_{1} c_{2} \eta^{\prime}
\end{array}\right)
$$

this employs the (generalized) Weyl curvature components $C \in \Omega^{2}\left(M, \mathfrak{s l}\left(\mathcal{E}^{\alpha}\right)\right)$ and $C^{\prime} \in$ $\mathcal{E}^{2}\left(\mathfrak{s l}\left(\mathcal{E}^{\alpha^{\prime}}\right)\right)$ and the generalized Cotton-York tensor $A \in \mathcal{E}^{2}\left(\mathcal{E}^{1}\right)$ (cf. [20]). Normality of the geometry and torsion-freeness imply that any possible trace of $C_{\gamma_{1}^{\prime} \gamma_{2}^{\prime} \eta}^{\gamma_{1} \gamma_{2} \varphi}, C_{\gamma_{1}^{\prime} \gamma_{2}^{\prime} \eta^{\prime}}^{\gamma_{1} \gamma_{2} \varphi^{\prime}}$ and $A_{\varphi^{\prime} \gamma_{1}^{\prime} \gamma_{2}^{\prime}}^{\varphi \gamma_{1} \gamma_{2}}$ vanishes.

5.3.2. Description of $D_{0}$ as the first $B G G$ operator. We consider the tractor bundle $V=\Lambda^{2} \mathcal{S}$, which under a choice of a Weyl connection $D$ decomposes according to

$$
[V]_{D}=\Lambda^{2}\left(\mathcal{E}^{\alpha} \oplus \mathcal{E}_{\alpha^{\prime}}\right)=\left(\begin{array}{c}
\mathcal{E}^{[\alpha \beta]} \\
\mathcal{E}^{\alpha \beta^{\prime}} \\
\mathcal{E}^{\left[\alpha^{\prime} \beta^{\prime}\right]}
\end{array}\right) .
$$

On the first chain spaces the Lie algebra differentials $\partial$ and $\partial^{*}$ are given as follows (indices within vertical bars are not included in the skew symmetrization):

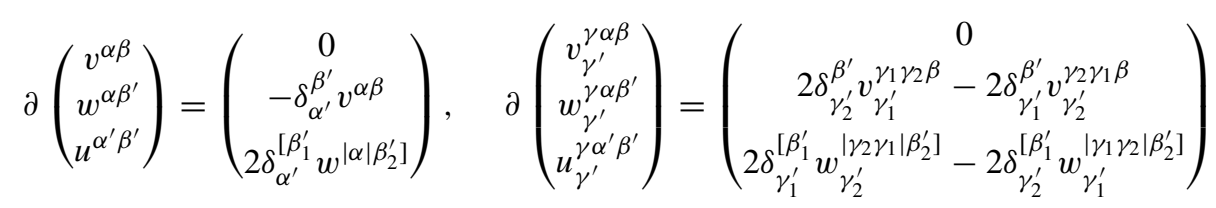




$$
\partial^{*}\left(\begin{array}{c}
v_{\gamma^{\prime}}^{\gamma \alpha \beta} \\
w_{\gamma^{\prime}}^{\gamma \alpha \beta^{\prime}} \\
u_{\gamma^{\prime}}^{\gamma \alpha^{\prime} \beta^{\prime}}
\end{array}\right)=\left(\begin{array}{c}
-2 w_{\tau^{\prime}}^{\left[\alpha_{1} \alpha_{2}\right] \tau^{\prime}} \\
u_{\tau^{\prime}}^{\alpha \tau^{\prime} \beta^{\prime}} \\
0
\end{array}\right), \quad \partial^{*}\left(\begin{array}{c}
v_{\gamma_{1}^{\prime} \gamma_{2}^{\prime}}^{\gamma_{1} \gamma_{2} \alpha \beta} \\
w_{\gamma_{1}^{\prime} \gamma_{2}^{\prime}}^{\gamma_{1} \gamma_{2} \alpha \beta^{\prime}} \\
u_{\gamma_{1}^{\prime} \gamma_{2}^{\prime} \gamma_{2}^{\prime} \alpha^{\prime}}^{\gamma^{\prime}}
\end{array}\right)=\left(\begin{array}{c}
2 w_{\gamma_{1}^{\prime} \tau^{\prime}}^{\gamma_{1}[\alpha \beta] \tau^{\prime}} \\
-u_{\gamma_{1}^{\prime} \tau^{\prime}}^{\gamma_{1} \alpha \tau^{\prime} \beta^{\prime}} \\
0
\end{array}\right)
$$

The Kostant Laplacian $\square=\partial \circ \partial^{*}+\partial^{*} \circ \partial$ acts on $[V]_{D}$ via

$$
\square\left(\begin{array}{c}
v^{\alpha \beta} \\
w^{\alpha \beta^{\prime}} \\
u^{\alpha^{\prime} \beta^{\prime}}
\end{array}\right)=\left(\begin{array}{c}
(2 q) v^{\alpha \beta} \\
(q-1) w^{\alpha \beta^{\prime}} \\
0
\end{array}\right) .
$$

The top slot of $\mathcal{E}^{1}(V)$ is $\mathcal{E}_{c}^{[\alpha \beta]}=\mathcal{E}_{\gamma^{\prime}}^{\gamma[\alpha \beta]}$ and coincides with the image of $\partial^{*}$. It is irreducible and the Kostant Laplacian acts by multiplication with $2(2 q-1)$. The middle slot of $\mathcal{E}^{1}(V)$, which is $\mathcal{E}_{c}^{\alpha \beta^{\prime}}$, decomposes into Im $\partial$, which are traces, and the trace-free part $\operatorname{Im} \partial^{*}=\mathcal{E}_{0 c}^{\alpha \beta^{\prime}}$. One finds that $\mathcal{E}_{0}{ }_{\gamma^{\prime}}^{\gamma \alpha \beta^{\prime}}=\mathcal{E}_{0}{ }_{\gamma^{\prime}}^{[\gamma \alpha] \beta^{\prime}} \oplus \mathcal{E}_{0}{ }_{\gamma^{\prime}}^{(\gamma \alpha) \beta^{\prime}}$ and $\square$ acts by $q$ on the alternating part and by $q-2$ on the symmetric part.

The tractor covariant derivative on $V$ is

$$
\left(\nabla^{\omega}\right)_{\gamma^{\prime}}^{\gamma}\left(\begin{array}{c}
v^{\alpha \beta} \\
w^{\alpha \beta^{\prime}} \\
u^{\alpha^{\prime} \beta^{\prime}}
\end{array}\right)=\left(\begin{array}{c}
D_{\gamma^{\prime}}^{\gamma} v^{\alpha \beta}+2 \mathrm{P}_{\gamma^{\prime} \tau^{\prime}}^{\gamma[\alpha} w^{\beta] \tau^{\prime}} \\
D_{\gamma^{\prime}}^{\gamma} w^{\alpha \beta^{\prime}}-\delta_{\gamma^{\prime}}^{\alpha} v^{\gamma \beta^{\prime}}+\mathrm{P}_{\gamma^{\prime} \tau^{\prime}}^{\gamma \alpha} u^{\beta^{\prime} \tau^{\prime}} \\
D_{\gamma^{\prime}}^{\gamma} u^{\alpha^{\prime} \beta^{\prime}}+2 \delta_{\gamma^{\prime}}^{\left[\alpha^{\prime}\right.} w^{\left.|\gamma| \beta^{\prime}\right]}
\end{array}\right)
$$

The first BGG splitting operator $L_{0}: \mathcal{E}^{\left(\alpha^{\prime} \beta^{\prime}\right)} \rightarrow \Gamma(V)$ is computed to be

$$
L_{0}\left(u^{\alpha^{\prime} \beta^{\prime}}\right)=\left(\begin{array}{c}
\frac{1}{2 q} \mathrm{P}_{\tau_{1}^{\prime} \tau_{2}^{\prime}}^{\alpha \beta} u^{\tau_{1}^{\prime} \tau_{2}^{\prime}}-\frac{1}{1-q} D_{\tau_{1}^{\prime}}^{[\alpha} D_{\tau_{2}^{\prime}}^{\beta]} u^{\tau_{1}^{\prime} \tau_{2}^{\prime}} \\
\frac{1}{1-q} D_{\tau^{\prime}}^{\alpha} u^{\tau^{\prime} \beta^{\prime}} \\
u^{\alpha^{\prime} \beta^{\prime}}
\end{array}\right)
$$

and the composition of $\nabla^{\omega} \circ L_{0}$ with the projection to the lowest slot is seen to yield our operator (14).

5.3.3. Prolongation of $D_{0}$. For a section $s$ of $V$ one first computes $K \bullet s \in \mathcal{E}^{2}(V)$, which is then mapped by $\partial^{*}$ into $\mathcal{E}^{1}(V)$,

$$
\partial^{*}\left(K \bullet\left(\begin{array}{c}
v^{\alpha \beta} \\
w^{\alpha \beta^{\prime}} \\
u^{\alpha^{\prime} \beta^{\prime}}
\end{array}\right)\right)=\left(\begin{array}{c}
2 C_{\gamma_{1}^{\prime} \varphi^{\prime} \eta}^{\gamma_{1}[\alpha \beta]} w^{\eta \varphi^{\prime}}+2 A_{\eta^{\prime} \varphi^{\prime}}^{[\alpha \mid \beta]} u^{\eta^{\prime} \varphi^{\prime}} \\
-2 C_{\gamma_{1}^{\prime} \varphi^{\prime} \eta^{\prime}}^{\gamma_{1} \alpha \beta^{\prime}} u^{\varphi^{\prime} \eta^{\prime}} \\
0
\end{array}\right)
$$

The first deformation map $\Phi_{1}$ is defined by $\Phi_{1}=-\square^{-1} \circ \partial^{*} \circ K \bullet$,

$$
\Phi_{1}\left(\left(\begin{array}{c}
0 \\
0 \\
u^{\alpha^{\prime} \beta^{\prime}}
\end{array}\right)\right)=\left(\begin{array}{c}
0 \\
\frac{2}{q} C_{\gamma_{1}^{\prime} \varphi^{\prime} \eta^{\prime}}^{\left[\gamma_{1} \alpha\right] \beta^{\prime}} u^{\varphi^{\prime} \eta^{\prime}}+\frac{2}{q-2} C_{\gamma_{1}^{\prime} p h^{\prime} \eta^{\prime}}^{\left(\gamma_{1} \alpha\right) \beta^{\prime}} u^{\varphi^{\prime} \eta^{\prime}} \\
0
\end{array}\right)
$$


Now we need to calculate $\partial^{*}$ of the change in curvature resulting from $\Phi_{1}$, which is just $\partial^{*} \circ d^{\nabla} \Phi_{1}$, since one quickly sees that $\partial^{*} \circ \Phi_{1\left[c_{1}\right.} \partial_{\left.c_{2}\right]}=0$. Both indices of a section $w^{\alpha \beta^{\prime}}$ are contracted into $C$ and the trace taken by $\partial^{*}$ vanishes by trace-freeness of $C, C^{\prime}$. Therefore we are only interested in the differential components of $d^{\nabla} \Phi_{1}$ given by

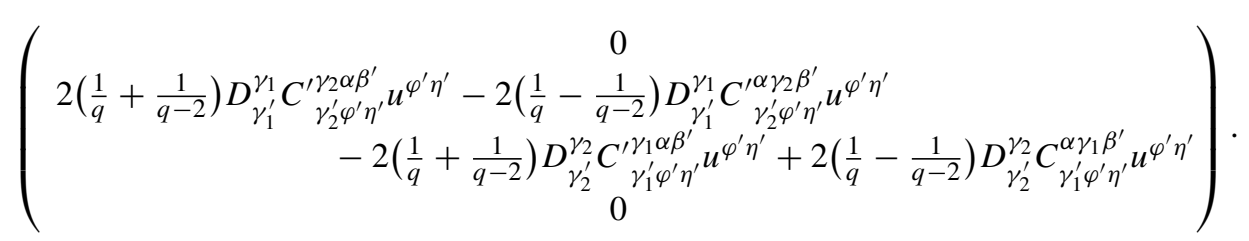

Applying $\partial^{*}$ we obtain the top slot contribution

$$
-4\left(\frac{1}{q}+\frac{1}{q-2}\right) D_{\tau^{\prime}}^{[\alpha} C_{\gamma_{1}^{\prime} \varphi^{\prime} \eta^{\prime}}^{\prime \prime \gamma_{1} \mid \beta \tau^{\prime}} u^{\varphi^{\prime} \eta^{\prime}}+4\left(\frac{1}{q}-\frac{1}{q-2}\right) D_{\tau^{\prime}}^{[\alpha} C_{\gamma_{1}^{\prime} \varphi^{\prime} \eta^{\prime}}^{\beta] \gamma_{1} \tau^{\prime}} u^{\varphi^{\prime} \eta^{\prime}}
$$

Adding the contributions of the top slot of (15) and (16) (after multiplication by $-\frac{1}{2(2 q-1)}$ ) to the modification map $\Phi_{1}$, we obtain the full modification map

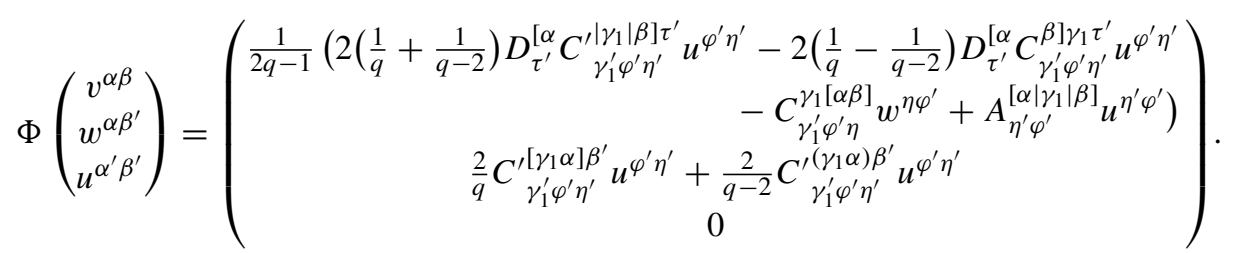

$\tilde{\nabla}=\nabla^{\omega}+\Phi$ is then the prolongation covariant derivative of the system $\left(D_{\gamma^{\prime}}^{\gamma} u^{\alpha^{\prime} \beta^{\prime}}\right)_{0}=0$.

\subsection{The case of infinitesimal automorphisms}

Let $\mathcal{A} M$ be the adjoint tractor bundle of a regular parabolic geometry $(\mathcal{G}, \omega)$ over $M$ and $\nabla^{\omega}$ the adjoint tractor covariant derivative. In [6] it was shown that parallel sections of the connection

$$
\tilde{\nabla} s=\nabla^{\omega} s+\kappa(\Pi(s), \cdot)
$$

are in one-to-one correspondence with infinitesimal automorphisms of $(\mathcal{G}, \omega)$, where $\Pi$ is the natural projection $\Pi: \mathcal{A} M \rightarrow T M$. This shows that it is of interest to consider the first BGG operator $\tilde{D}_{0}$ obtained from $\tilde{\nabla}$. If the parabolic geometry $(\mathcal{G}, \omega)$ is normal, the curvature of $\tilde{\nabla}$ lies in the kernel of $\partial_{\mathcal{A} M}^{*}$. Therefore, exactly as in Corollary 3.1, one sees that $\Pi_{0}: \mathcal{A} M \rightarrow H_{0}$ and $\tilde{L}_{0}: H_{0} \rightarrow \mathcal{A M}$ are inverse isomorphisms between the space of parallel sections of $\tilde{\nabla}$ and the kernel of $\tilde{D}_{0}$. Thus, the operator $\tilde{D}_{0}$ describes the infinitesimal automorphisms of $(\mathcal{G}, \omega)$ and is automatically prolonged by $\tilde{\nabla}$.

It is shown that if the parabolic geometry is also torsion-free or 1-graded, one has $\partial_{\mathcal{A} M}^{*} \kappa=0$, i.e., for every $s \in \mathcal{A} M$ one has $\partial_{\mathcal{A} M}^{*} \kappa(\Pi(s), \cdot)=0$. But in the torsionfree case, the map $\xi \mapsto \kappa(\Pi(s), \xi)$ is evidently homogeneous of degree $\geq 1$. Therefore, 
if we know that $H_{1}\left(\mathfrak{g}_{-}, \mathfrak{g}\right)$ sits in homogeneity $\leq 0$, we see that $\xi \mapsto \kappa(\Pi(s), \xi)$ lies in $\operatorname{Im} \partial_{\mathcal{A} M}^{*}$.

Thus we have:

Theorem 5.1. Let $(\mathcal{G} \rightarrow M, \omega)$ be a torsion-free, normal parabolic geometry with $H_{1}\left(\mathfrak{g}_{-}, \mathfrak{g}\right)$ concentrated in homogeneity $\leq 0$. Then $\tilde{\nabla}$ from (17) coincides with the prolongation covariant derivative on $\mathcal{A} M$. In particular, the usual first $B G G$ operator $D_{0}$ coincides with $\tilde{D}_{0}$ and thus describes infinitesimal automorphisms.

We note that the homogeneity condition on $H_{1}\left(\mathfrak{g}_{-}, \mathfrak{g}\right)$ is satisfied for all parabolic geometries of type $(G, P)$ with $\mathfrak{g}$ simple and $(G, P)$ not corresponding to projective structures or contact projective structures.

Acknowledgments. The results presented in the paper are inspired very much by a construction devised by A. Čap to describe infinitesimal automorphisms for parabolic geometries. The authors would like to thank him for a lot of helpful discussions. The first author was supported by the IK I008-N funded by the University of Vienna and project P 19500-N13 of the "Fonds zur Förderung der wissenschaftlichen Forschung" (FWF). The second and the third authors were supported by the institutional grant MSM 0021620839 and by the grant GA CR 201/08/397. The last author was supported from Basic Research Center no. LC505 (Eduard Čech Center for Algebra and Geometry) of the Ministry of Education of the Czech Republic.

\section{References}

[1] Bailey, T. N., Eastwood, M. G., Gover, A. R.: Thomas's structure bundle for conformal, projective and related structures. Rocky Mountain J. Math. 24, 1191-1217 (1994) Zbl 0828.53012 MR 1322223

[2] Baum, H., Friedrich, T., Grunewald, R., Kath, I.: Twistor and Killing Spinors on Riemannian Manifolds. Seminarberichte 108, Humboldt Universität, Sektion Mathematik, Berlin (1990) Zbl 0705.53004 MR 1084369

[3] Branson, T.: Conformal structure and spin geometry. In: Dirac Operators: Yesterday and Yoday, Int. Press, Sommerville, MA, 163-191 (2005) Zbl 1109.53051 MR 2205371

[4] Branson, T., Čap, A., Eastwood, M. G., Gover, A. R.: Prolongations of geometric overdetermined systems. Int. J. Math. 17, 641-664 (2006) Zbl 1101.35060 MR 2246885

[5] Calderbank, D., Diemer, T.: Differential invariants and curved Bernstein-Gelfand-Gelfand sequences. J. Reine Angew. Math. 537, 67-103 (2001) Zbl 0985.58002 MR 1856258

[6] Čap, A.: Infinitesimal automorphisms and deformations of parabolic geometries. J. Eur. Math. Soc. 10, 415-437 (2008) Zbl 1161.32020 MR 2390330

[7] Čap, A., Slovák, J.: Weyl structures for parabolic geometries. Math. Scand. 93, 53-90 (2003) Zbl 1076.53029 MR 1997873

[8] Čap, A., Slovák, J.: Parabolic Geometries I: Background and General Theory. Math. Surveys Monogr. 154, Amer. Math. Soc. (2009) Zbl 1183.53002 MR 2532439

[9] Čap, A., Slovák, J., Souček, V.: Bernstein-Gelfand-Gelfand sequences. Ann. of Math. 154, 97-113 (2001) Zbl 1159.58309 MR 1847589

[10] Cartan, É.: Les espaces à connexion conforme. Ann. Soc. Polon. Math. 2, 171-221 (1923) JFM 50.0493.01

[11] Cartan, É.: Sur les variétés à connexion projective. Bull. Soc. Math. France 52, 205-241 (1924) JFM 50.0500.02 MR 1504846 
[12] Chern, S. S., Moser, J. K.: Real hypersurfaces in complex manifolds. Acta Math. 133, 219271 (1974) Zbl 0302.32015 MR 0709144

[13] Dunajski, M., Tod, P.: Four dimensional metrics conformal to Kähler. Math. Proc. Cambridge Philos. Soc. 148, 485-503 (2010) Zbl 1188.53078 MR 2609304

[14] Eastwood, M. G.: Notes on conformal differential geometry. Suppl. Rend. Circ. Mat. Palermo 43, 57-76 (1996) Zbl 0911.53020 MR 1463509

[15] Eastwood, M. G.: Notes on projective differential geometry. In: Symmetries and Overdetermined Systems of Partial Differential Equations, IMA Vol. Math. Appl. 144, Springer, New York, 41-60 (2008) Zbl 1186.53020 MR 2384705

[16] Eastwood, M. G., Gover, A. R.: Prolongation on contact manifolds. arXiv:0910.5519

[17] Eastwood, M. G., Matveev, V.: Metric connections in projective differential geometry. In: Symmetries and Overdetermined Systems of Partial Differential Equations, IMA Vol. Math. Appl. 144, Springer, New York, 339-351 (2008) Zbl 1144.53027 MR 2384718

[18] Gover, A. R.: Almost Einstein and Poincaré-Einstein manifolds in Riemannian signature. J. Geom. Phys. 60, 182-204 (2010) Zbl 1194.53038 MR 2587388

[19] Gover, A. R., Šilhan, J.: The conformal Killing equation on forms-prolongations and applications, Differential Geom. Appl. 26, 244-266 (2008) Zbl 1144.53036 MR 2421703

[20] Gover, A. R., Slovák, J.: Invariant local twistor calculus for quaternionic structures and related geometries. J. Geom. Phys. 32, 14-56 (1999) Zbl 0981.53031 MR 1723137

[21] Gover, A. R., Somberg, P., Souček, V.: Yang-Mills detour complexes and conformal geometry. Comm. Math. Phys. 278, 307-327 (2008) Zbl 1141.58013 MR 2372762

[22] Hammerl, M.: Invariant prolongation of BGG-operators in conformal geometry. Arch. Math. (Brno) 44, 367-384 (2008) Zbl 1212.53014 MR 2501573

[23] Hammerl, M.: Natural prolongations of BGG operators. Thesis, Univ. of Vienna (2009)

[24] Hammerl, M., Somberg, P., Souček, V., Šilhan, J.: Invariant prolongation of overdetermined PDEs in projective, conformal, and Grassmannian geometry. Ann. Global Anal. Geom. 42, 121-145 (2012) Zbl pre06037517

[25] Kostant, B.: Lie algebra cohomology and the generalized Borel-Weil theorem. Ann. of Math. (2) 74, 329-387 (1961) Zbl 0134.03501 MR 0142696

[26] Leitner, F.: Conformal Killing forms with normalisation condition. Suppl. Rend. Circ. Mat. Palermo (2) 75, 279-292 (2005) Zbl 1101.53040 MR 2152367

[27] Morimoto, T.: Lie algebras, geometric structures and differential equations on filtered manifolds. In: Lie Groups, Geometric Structures and Differential Equations-One Hundred Years after Sophus Lie (Kyoto/Nara, 1999), Adv. Stud. Pure Math. 37, Math. Soc. Japan, Tokyo, 205-252 (2002) Zbl 1048.58015 MR 1980903

[28] Neusser, K.: Prolongation on regular infinitesimal flag manifolds. Int. J. Math. 23, no. 4, art. ID 1250007, 41 pp. (2012) Zbl pre06026470

[29] Penrose, R., Rindler, W.: Spinors and Space-Time Vols. 1, 2, Cambridge Univ. Press (1984, 1986) Zbl 0538.53024(Vol. 1) Zbl 0591.53022(Vol. 2) MR 0776784(Vol. 1) MR 0838301(Vol. 2)

[30] Semmelmann, U.: Conformal Killing forms on Riemannian manifolds. Math. Z. 245, 503527 (2003) Zbl 1061.53033 MR 2021568

[31] Sharpe, R. W.: Differential Geometry: Cartan's Generalization of Klein's Erlangen Program. Grad. Texts in Math. 166, Springer (1997) Zbl 0876.53001 MR 1453120

[32] Spencer, D. C.: Overdetermined systems of linear partial differential equations. Bull. Amer. Math. Soc. 75, 179-239 (1969) Zbl 0185.33801 MR 0242200 